\title{
The Einstein@Home Gamma-ray Pulsar Survey. II. Source Selection, Spectral Analysis, and Multiwavelength Follow-up
}

\author{
J. Wu ${ }^{1}$ (D) C. J. Clark ${ }^{2,3}$ (D), H. J. Pletsch ${ }^{2,3}$ (D), L. Guillemot ${ }^{1,4,5}$ (D), T. J. Johnson ${ }^{6}$, P. Torne ${ }^{1}$, D. J. Champion ${ }^{1}$ (D), J. Deneva ${ }^{6}$ (D), \\ P. S. Ray ${ }^{7}$ (i) D. Salvetti ${ }^{8}$, M. Kramer ${ }^{1,9,10}$ (D), C. Aulbert ${ }^{2,3}$ (D), C. Beer ${ }^{2,3}$ (D), B. Bhattacharyya ${ }^{11}$, O. Bock ${ }^{2,3}$, F. Camilo ${ }^{12}$ (iD), \\ I. Cognard $^{4,5}$, A. Cuéllar ${ }^{2,3}$, H. B. Eggenstein ${ }^{2,3}$ (D) H. Fehrmann ${ }^{2,3}$, E. C. Ferrara ${ }^{13}$, M. Kerr ${ }^{7}$ (D) B. Machenschalk ${ }^{2,3}$, \\ S. M. Ransom ${ }^{14}$ (D), S. Sanpa-Arsa ${ }^{15}$, and K. Wood ${ }^{16}$ \\ ${ }^{1}$ Max-Planck-Institut für Radioastronomie, Auf dem Hügel 69, D-53121 Bonn, Germany; jwu@mpifr-bonn.mpg.de, lucas.guillemot@cnrs-orleans.fr \\ 2 Albert-Einstein-Institut, Max-Planck-Institut für Gravitationsphysik, D-30167 Hannover, Germany \\ ${ }^{3}$ Leibniz Universität Hannover, D-30167 Hannover, Germany \\ ${ }^{4}$ Laboratoire de Physique et Chimie de l'Environnement et de l'Espace-Université d'Orléans/CNRS, F-45071 Orléans Cedex 02, France \\ ${ }^{5}$ Station de radioastronomie de Nançay, Observatoire de Paris, CNRS/INSU, F-18330 Nançay, France \\ ${ }^{6}$ College of Science, George Mason University, Fairfax, VA 22030, resident at Naval Research Laboratory, Washington, DC 20375, USA \\ ${ }^{7}$ Space Science Division, Naval Research Laboratory, Washington, DC 20375-5352, USA \\ ${ }^{8}$ INAF-Istituto di Astrofisica Spaziale e Fisica Cosmica Milano, via E. Bassini 15, I-20133 Milano, Italy \\ ${ }^{9}$ Jodrell Bank Centre for Astrophysics, School of Physics and Astronomy, The University of Manchester, M13 9PL, UK \\ ${ }^{10}$ University of Manchester, Manchester, M13 9PL, UK \\ ${ }^{11}$ National Centre for Radio Astrophysics, Tata Institute of Fundamental Research, Pune 411 007, India \\ ${ }^{12}$ Square Kilometre Array South Africa, Pinelands, 7405, South Africa \\ ${ }^{13}$ NASA Goddard Space Flight Center, Greenbelt, MD 20771, USA \\ ${ }^{14}$ National Radio Astronomy Observatory, Charlottesville, VA 22903, USA \\ ${ }^{15}$ National Astronomical Research Institute of Thailand, 191 Siriphanich Bidg., 2nd Fl., Huay Kaew Rd., Suthep District, Muang, Chiang Mai 50200, Thailand \\ ${ }^{16}$ Praxis Inc., Alexandria, VA 22303, resident at Naval Research Laboratory, Washington, DC 20375, USA \\ Received 2017 September 13; revised 2017 November 29; accepted 2017 December 12; published 2018 February 15
}

\begin{abstract}
We report on the analysis of 13 gamma-ray pulsars discovered in the Einstein@Home blind search survey using Fermi Large Area Telescope (LAT) Pass 8 data. The 13 new gamma-ray pulsars were discovered by searching 118 unassociated LAT sources from the third LAT source catalog (3FGL), selected using the Gaussian Mixture Model machine-learning algorithm on the basis of their gamma-ray emission properties being suggestive of pulsar magnetospheric emission. The new gamma-ray pulsars have pulse profiles and spectral properties similar to those of previously detected young gamma-ray pulsars. Follow-up radio observations have revealed faint radio pulsations from two of the newly discovered pulsars and enabled us to derive upper limits on the radio emission from the others, demonstrating that they are likely radio-quiet gamma-ray pulsars. We also present results from modeling the gamma-ray pulse profiles and radio profiles, if available, using different geometric emission models of pulsars. The high discovery rate of this survey, despite the increasing difficulty of blind pulsar searches in gamma rays, suggests that new systematic surveys such as presented in this article should be continued when new LAT source catalogs become available.
\end{abstract}

Key words: gamma rays: stars - pulsars: individual (PSR J0002+6216, PSR J0631+0646, PSR J1624-4041, PSR $\mathrm{J} 2017+3625)$

Supporting material: machine-readable table

\section{Introduction}

Pulsars are rapidly rotating neutron stars with rotational periods ranging from more than $10 \mathrm{~s}$ to just a few milliseconds. Since their discovery in 1967 (Hewish et al. 1968), various pulsar surveys have discovered over 2600 pulsars. ${ }^{17}$ While the large majority of the known pulsars have been detected in the radio, pulsars are occasionally detected at optical, infrared, UV, $\mathrm{X}$-ray, or even gamma-ray frequencies, enabling multiwavelength studies (for recent examples see Mignani et al. 2017; Swiggum et al. 2017).

During the first $8 \mathrm{yr}$ of operation, over 200 gamma-ray pulsars have been detected by the Fermi Large Area Telescope $^{18}$ (LAT; Atwood et al. 2009). The majority of the detected gamma-ray pulsars were first found in radio, discovered from either radio pulsar surveys or targeted radio

\footnotetext{
17 http://www.atnf.csiro.au/research/pulsar/psrcat/

${ }^{18}$ See https://tinyurl.com/fermipulsars for the list of LAT-detected pulsars.
}

observations of unassociated LAT sources (i.e., sources with no obvious counterparts at other wavelengths; see, e.g., Cognard et al. 2011; Keith et al. 2011; Ransom et al. 2011; Camilo et al. 2012, 2015; Guillemot et al. 2012a; Kerr et al. 2012; Barr et al. 2013; Bhattacharyya et al. 2013; Cromartie et al. 2016). However, a substantial fraction of the gamma-ray pulsars have been discovered by direct, blind searches of the LAT data (e.g., Abdo et al. 2009; Saz Parkinson et al. 2010; Pletsch et al. 2012a; Clark et al. 2015).

Gamma-ray pulsars found in blind searches are interesting for many reasons. These pulsars are young and energetic with characteristic ages $<3 \mathrm{Myr}$ and spin-down power $\dot{E}>10^{33} \mathrm{erg}$ $\mathrm{s}^{-1}$ (see Figure 1 of the second Fermi LAT catalog of gammaray pulsars, hereafter 2PC; Abdo et al. 2013). These young energetic pulsars often have timing noise and glitches. This absence of timing coherence makes their pulsations more difficult to find in the low count-rate gamma-ray data acquired over time spans of years. The discovery of PSR J1906+0722 
(Clark et al. 2015) demonstrated the ability of the improved semicoherent blind search technique to detect pulsars even when the data contain timing noise and a substantial glitch. Such blind search methods can reduce the bias against the discovery of young and energetic radio-quiet pulsars in the current pulsar population.

Although the 41 pulsars found in previous blind gamma-ray searches represent a small fraction of the total pulsar population, this increasing population forms a very distinct group with extremely faint or undetectable radio emission. Besides the possible detections of J1732-3131 (Maan et al. 2012) and Geminga (Maan 2015), only four gammaray-discovered pulsars have also been detected in radio, two of them being radio-loud (we follow the convention used in 2PC, i.e., pulsars are considered radio-quiet if their radio flux densities at $1400 \mathrm{MHz}, S_{1400}$, are smaller than $30 \mu \mathrm{Jy}$ ), J1741 -2054 and $\mathrm{J} 2032+4127$ (Camilo et al. 2009), and the remaining two, J0106+4855 (Pletsch et al. 2012a) and J1907 +0602 (Abdo et al. 2010), being considered radio-quiet.

To further increase the number of known gamma-ray pulsars, a new blind search of unidentified LAT sources with gamma-ray emission properties resembling known pulsars was initiated. This search has been conducted on the distributed volunteer computing system Einstein@Home ${ }^{19}$ using the newly improved Pass 8 LAT data. This data set provides a number of improvements such as better energy reconstruction and better background rejection (see Atwood et al. 2013), therefore increasing its sensitivity.

Based on their gamma-ray properties, we have selected and searched 118 unassociated LAT sources, resulting in the discovery of 17 pulsars. The results of this search are presented in two papers: Clark et al. (2017, hereafter Paper I) focused on the search method, sensitivity, and temporal characteristics of the recent pulsar discoveries. In this second paper, we present the source selection scheme, the data preparation process, and detailed gamma-ray analyses and radio follow-up observations of the discoveries. In Section 2, we describe the method used for selecting gamma-ray sources for the blind search. Section 3 describes the analysis procedure we followed for preparing the gamma-ray data to be searched for pulsars. Gamma-ray, X-ray, and radio follow-up analyses of the newly discovered pulsars are described in Section 4, and we conclude with a discussion of the properties of the new pulsars.

\section{Source Selection}

\subsection{FGL Catalog}

The third catalog of LAT sources (hereafter 3FGL; Acero et al. 2015) lists the properties of 3033 gamma-ray sources detected by the LAT in the first $4 \mathrm{yr}$ of data taking. More than $30 \%$ of the 3FGL sources were unassociated at the time of publication. More than 100 of these unassociated sources have been demonstrated to be previously unknown pulsars, discovered either in deep targeted radio observations or in blind searches using the LAT data. Due to the observing time and processing resources required for a timing search, identifying which of these sources are most likely to be pulsars has become a task of paramount importance. In contrast to several other classes of gamma-ray sources, pulsars have significant cutoffs in their emission spectra at energies of a few

\footnotetext{
19 https://einsteinathome.org
}

$\mathrm{GeV}$ and gamma-ray fluxes that are generally very stable (however, see Allafort et al. 2013, for a counterexample); hence, the curvature significance ${ }^{20}$ ("Signif_Curve", $S_{c}$ ) and the variability index ${ }^{21}$ ("Variability_Index", VI), which are respectively measures of the curvature of a source's spectrum and of its gamma-ray flux variability, have been successfully applied in previous similar surveys (e.g., Pletsch et al. 2012a).

We note that only a preliminary version of the 3FGL catalog was available when our survey was initiated. We therefore assessed the pulsar likelihood of the unassociated sources from this preliminary catalog. As a cross-check of our source selection results, we have compared the data from the preliminary catalog with those from 3FGL, finding differences in one specific parameter only. These differences are discussed in the next section.

\subsection{Pulsar Candidate Selection}

Although using $S_{c}$ and the VI seems to be enough to identify pulsar candidates, extra care needs to be taken, as these two parameters are correlated with the detection significance. A number of groups have developed different schemes for classifying sources, involving machine-learning techniques (Lee et al. 2012; Mirabal et al. 2012; Saz Parkinson et al. 2016). In particular, Lee et al. (2012) have shown that including the gamma-ray flux as a third dimension in the pulsar classification scheme can directly correct the abovementioned correlation. Applying the Gaussian Mixture Model (GMM) classification scheme from Lee et al. (2012), we used the VI, $S_{c}$, and $F_{1000}$ (gamma-ray flux above $1 \mathrm{GeV}$ ) parameters from the catalog to calculate the pulsar likelihood $R_{s}$ for all the sources. A positive $\log R_{s}$ indicates that the source is likely to be a pulsar (see Lee et al. 2012, for a detailed discussion). A list of 341 sources with positive logarithmic pulsar likelihood $\left(\log R_{s}\right)$ values and no firm associations with any other astrophysical sources was obtained.

As mentioned in Section 2.1, the list of pulsar candidates was produced by analyzing a preliminary version of the 3FGL catalog. We verified that the characteristics of most of the sources from the preliminary catalog are identical to those from the final catalog. One difference concerns the definition of the spectral curvature Test Statistic (TS), $\mathrm{TS}_{\text {curve }}$, listed instead of the curvature significance in the preliminary version of the catalog, $S_{c}=\sqrt{\mathrm{TS}_{\text {curve }} \times R_{\text {syst }}}$, where $R_{\text {syst }}$ accounts for systematic uncertainties in the effective area. We verified that using $\mathrm{TS}_{\text {curve }}$ instead of $S_{c}$ as one of the inputs of the GMM does not affect our classification results.

\section{Data Preparation}

\subsection{The Spectral Analysis Pipeline}

One of the main difficulties in blind searches for gamma-ray pulsars is separating background emission from photons originating from the sources of interest. Due to the wide and energydependent point-spread function of the LAT at low energies, ${ }^{22}$ neighboring sources within a few degrees of a given direction can

\footnotetext{
${ }^{20}$ Significance (in $\sigma$ units) of the fit improvement when assuming a curved spectral type (e.g., PLEC; see Section 3.1) instead of a simple power law for the source of interest. Values greater than 4 indicate significant curvature.

21 Index quantifying the variability of a source on a timescale of months. An index larger than 72.44 corresponds to a $>99 \%$ confidence probability that the source of interest has a variable flux.

22 https://www.slac.stanford.edu/exp/glast/groups/canda/lat_Performance.htm
} 
raise the background level in the data set considered for the search. In the past, blind searches often adopted a so-called "cookie cutter" to select photons and increase the signal-to-noise ratio, i.e., they restricted the region of interest (ROI) by selecting events with reconstructed directions found within, say, $\sim 1^{\circ}$ of the considered sky location. Although this technique can efficiently separate source and background photons for some bright pulsars or pulsars in regions of low background contamination, most of the young gamma-ray pulsars are located near the Galactic plane, where the diffuse background emission is strong and where the effectiveness of the cookie cutter selection method decreases. Kerr (2011) mitigated this problem by proposing a photon-weighting technique, which uses information about the spectrum of the targeted source and the instrumental response of the LAT. Probabilities that photons originate from the source can then be calculated, relaxing the need to select narrow sky regions and greatly improving our sensitivity to weak periodic signals.

Consequently, accurately determining the spectra of the sources we want to search for pulsations is key for calculating photon weights and thereby increasing the signal-to-noise ratio. We assembled a spectral analysis pipeline based on the Pointlike analysis package (Kerr 2010), allowing us to derive the spectral parameters of the search targets and to assign good photon weights for the selected data sets. We initially considered LAT data recorded between 2008 August 4 and 2014 April 6 for our survey and included photons recorded until 2015 July 7 after a few tens of sources had been searched (see Section 3.2). We used the Fermi Science Tools ${ }^{23}$ to extract Pass 8 Source class events, processed with the P8_SOURCE_V3 instrument response functions (IRFs). The Science Tools, IRFs, and models for the Galactic and extragalactic diffuse gamma-ray emission used here are internal pre-release versions of the Pass 8 data analysis, which were the latest versions available to us when the survey began. The differences in the best-fit parameters are marginal, compared to the analysis with the most recent IRFs. Therefore, the weights as calculated with the old IRFs are also very similar. Specifications of follow-up data analyses are given in Section 4. We used gtselect to select photons with reconstructed directions within $8^{\circ}$ of the 3 FGL positions, photon energies $>100 \mathrm{MeV}$, and zenith angles $<100^{\circ}$. We only included photons detected when the LAT was operating in normal science mode and when the rocking angle of the spacecraft was less than $52^{\circ}$. Photons were then binned into 30 logarithmically spaced energy bins, with a spatial bin size of $00^{\circ} 1$.

For each 3FGL target, a spectral model for the sources within the corresponding ROIs was constructed by including all 3FGL sources within $13^{\circ}$. Spectral parameters of point sources within $5^{\circ}$ were allowed to vary. A binned maximum likelihood analysis was performed to measure the gamma-ray spectra of the targeted sources, which were modeled with exponentially cutoff power laws ("PLEC" spectral shapes) of the form

$$
\frac{d N}{d E}=K\left(\frac{E}{1 \mathrm{GeV}}\right)^{-\Gamma} \exp \left(-\frac{E}{E_{\mathrm{cut}}}\right),
$$

where $K$ is a normalization factor, $E_{\text {cut }}$ is the cutoff energy, and $\Gamma$ is the photon index. The above expression accurately reproduces the phase-averaged spectral properties of the majority of known gamma-ray pulsars (see, e.g., 2PC). The

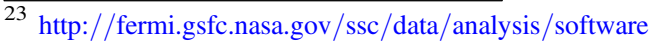

normalization parameters of the Galactic diffuse emission and the isotropic diffuse background components were left free in the fits. The best-fit source models from the likelihood analysis with Pointlike were used as inputs for gtsrcprob, to determine the probabilities that the selected photons were indeed emitted by our targets.

In order to verify the goodness of the fits and check for possible issues in the likelihood results, we produced source significance TS maps and plots of the spectral energy distribution (SED) for each analyzed source. For some of the sources, the best-fit cutoff energies were suspiciously high and were in particular much higher than those of known gamma-ray pulsars. These sources have spectra with low curvature and could potentially be associated with supernova remnants (SNRs) or pulsar wind nebulae (PWNe), which are known to have harder spectra than pulsars. For some sources a very high cutoff energy close to the upper bound of $1 \mathrm{TeV}$ used for the fit was found, suggesting low spectral curvature. In some cases the best-fit photon index $\Gamma$ was close to 0 . These low photon indices were found for sources with low TS values. We flagged these problematic sources, but we included them in the survey despite the abnormal spectral results since we may still be able to detect pulsations from these sources. We note that SEDs for the latter sources were generally consistent with 3FGL results. In addition, for a small number of 3FGL sources our analysis failed to converge, possibly because of complicated sky regions. Those sources were removed from the target list and will be revisited in the future. As a result, the original target list was trimmed down to 118 sources, which are listed in Table 1.

We eventually obtained data sets consisting of lists of photon arrival times to be searched for pulsations, photon weights, and spacecraft positions calculated at each photon time, which are necessary to correct the arrival times for Doppler shifts caused by the motion of the telescope with respect to the sources. These data sets were then passed to the blind search algorithm, for searching for new pulsars among our target sources.

\subsection{Relocalization}

Following the first few discoveries (summarized in Section 3.3), we noticed that the timing positions of a few pulsars (see Paper I for the timing positions of the discovered pulsars) were well outside the $95 \%$ confidence regions from the 3FGL catalog. The observed discrepancies could be caused by the fact that 3FGL catalog positions were determined using $4 \mathrm{yr}$ of Pass 7 reprocessed data, while we used 5.5 yr of Pass 8 data, which have higher angular resolution. To mitigate this discrepancy, we relocalized the sources using Pointlike, by varying the sky coordinates of the sources until the maximum likelihood was found. The results of the relocalization analysis for the new pulsars are given in Table 2.

In most cases, the relocalized positions are closer to the pulsar timing positions than the catalog ones. In addition, the 95\% semimajor axes of the relocalized positions are smaller than in 3FGL. Although this implies a smaller number of trials in sky position for the blind search, leading to a greatly reduced overall computational cost, the true pulsar positions may still fall outside of the error ellipses. In some cases, the timing position is found to be out of both the 3FGL error ellipse and the ellipse from our analysis. From the 47th source onward, we therefore adopted the relocalized positions with three times the 
Table 1

Ranked Source List

\begin{tabular}{|c|c|c|c|c|c|c|c|c|c|c|c|}
\hline 3FGL Name & $\begin{array}{l}\text { Searched R.A. } \\
\quad(\mathrm{J} 2000)\end{array}$ & $\begin{array}{l}\text { Searched Decl. } \\
\text { (J2000) }\end{array}$ & $\begin{array}{l}\text { Search Radius } \\
\quad(\operatorname{arcmin})\end{array}$ & VI & $\mathrm{TS}_{\text {curve }}$ & $\mathrm{TS}_{\mathrm{cut}}$ & $\begin{array}{l}E_{\text {cut }} \\
(\mathrm{GeV})\end{array}$ & $\Gamma$ & TS & $\log R_{S}$ & Class \\
\hline $\mathrm{J} 1745.3-2903 \mathrm{c}$ & $17: 45: 22.32$ & $-29: 03: 46.80$ & 2.05 & 48.42 & 275.2 & 378.7 & 2.2 & 1.4 & 3407 & 18.85 & $\cdots$ \\
\hline $\mathrm{J} 1746.3-2851 \mathrm{c}$ & $17: 46: 22.51$ & $-28: 51: 45.72$ & 2.12 & 57.06 & 113.0 & 364.7 & 4.0 & 1.5 & 2373 & 14.31 & pwn \\
\hline J2017.9+3627 & $20: 17: 56.33$ & $+36: 27: 32.76$ & 3.10 & 39.86 & 179.3 & 198.9 & 1.9 & 1.4 & 1876 & 13.61 & $\ldots$ \\
\hline $\mathrm{J} 1839.3-0552^{\dagger}$ & $18: 39: 23.52$ & $-05: 52: 53.76$ & 3.07 & 37.43 & 83.7 & 135.1 & 2.3 & 1.2 & 714 & 13.26 & $\cdots$ \\
\hline J1906.6+0720 ${ }^{\dagger}$ & 19:06:41.14 & $+07: 20: 02.04$ & 3.33 & 41.70 & 87.9 & 68.6 & 7.0 & 2.0 & 1580 & 12.51 & $\cdots$ \\
\hline $\mathrm{J} 1910.9+0906^{\dagger}$ & 19:10:58.61 & $+09: 06: 01.80$ & 1.55 & 52.13 & 53.2 & 17.4 & 41.7 & 2.1 & 4790 & 12.31 & snr \\
\hline $\mathrm{J} 1636.2-4734^{\dagger}$ & $16: 36: 16.49$ & $-47: 34: 49.08$ & 4.58 & 54.63 & 106.0 & 47.1 & 7.1 & 1.9 & 1180 & 12.28 & snr \\
\hline J1848.4-0141 & $18: 48: 28.39$ & $-01: 41: 33.72$ & 7.27 & 52.63 & 109.0 & 13.8 & 9.8 & 2.5 & 1457 & 11.81 & $\ldots$ \\
\hline $\mathrm{J} 1405.4-6119^{\dagger}$ & $14: 05: 25.46$ & $-61: 19: 00.48$ & 2.83 & 43.93 & 61.1 & 19.7 & 8.2 & 2.1 & 1671 & 11.39 & $\ldots$ \\
\hline $\mathrm{J} 1111.9-6038^{\dagger}$ & $11: 11: 58.44$ & $-60: 38: 27.96$ & 1.96 & 46.69 & 81.4 & 58.5 & 10.4 & 1.9 & 3624 & 11.36 & spp \\
\hline $\mathrm{J} 1748.3-2815 \mathrm{c}$ & $17: 48: 22.20$ & $-28: 15: 32.04$ & 2.73 & 34.06 & 77.4 & 68.6 & 4.7 & 1.4 & 489 & 11.26 & $\cdots$ \\
\hline J1622.9-5004 & $16: 22: 54.31$ & $-50: 04: 31.08$ & 2.17 & 54.35 & 72.4 & 73.3 & 8.0 & 1.6 & 891 & 10.21 & $\cdots$ \\
\hline $\mathrm{J} 0223.6+6204^{\dagger}$ & $02: 23: 37.46$ & $+62: 04: 51.96$ & 3.51 & 41.77 & 86.3 & 182.6 & 1.8 & 1.5 & 1089 & 9.78 & $\cdots$ \\
\hline $\mathrm{J} 1823.2-1339^{\dagger}$ & $18: 23: 16.90$ & $-13: 39: 04.68$ & 2.60 & 47.54 & 29.7 & 47.4 & 9.0 & 1.9 & 1004 & 9.72 & $\cdots$ \\
\hline $\mathrm{J} 1745.1-3011$ & $17: 45: 11.30$ & $-30: 11: 57.84$ & 6.17 & 59.68 & 92.7 & 88.8 & 0.6 & 0.4 & 459 & 9.69 & spp \\
\hline $\mathrm{J} 1800.8-2402^{\dagger}$ & 18:00:53.18 & $-24: 02: 06.36$ & 3.13 & 46.65 & 36.4 & 21.3 & 11.3 & 1.7 & 575 & 9.69 & $\cdots$ \\
\hline J1749.2-2911 & $17: 49: 15.58$ & $-29: 11: 34.44$ & 7.21 & 41.77 & 50.9 & 43.6 & 1.6 & 1.3 & 265 & 9.62 & $\cdots$ \\
\hline $\mathrm{J} 1306.4-6043^{\dagger}$ & $13: 06: 27.50$ & $-60: 43: 54.12$ & 2.48 & 35.69 & 65.9 & 42.6 & 8.6 & 1.7 & 1108 & 9.59 & $\ldots$ \\
\hline J1104.9-6036 ${ }^{\dagger}$ & 11:04:59.42 & $-60: 36: 32.76$ & 4.10 & 43.09 & 77.4 & 64.6 & 3.6 & 1.7 & 769 & 9.42 & $\ldots$ \\
\hline J0634.1+0424 & $06: 34: 06.79$ & $+04: 24: 22.32$ & 9.77 & 42.87 & 123.3 & 60.2 & 1.8 & 2.2 & 1421 & 9.41 & $\cdots$ \\
\hline $\mathrm{J} 1552.8-5330$ & $15: 52: 50.90$ & $-53: 30: 47.16$ & 6.98 & 46.44 & 56.6 & 50.3 & 1.8 & 1.0 & 210 & 9.26 & $\cdots$ \\
\hline $\mathrm{J} 1747.0-2828^{\dagger}$ & $17: 47: 05.98$ & $-28: 28: 54.84$ & 3.65 & 90.61 & 159.7 & 135.3 & 2.5 & 1.8 & 1676 & 9.22 & $\cdots$ \\
\hline J1650.3-4600 & $16: 50: 23.76$ & $-46: 00: 50.76$ & 3.14 & 55.06 & 54.6 & 55.0 & 4.8 & 1.8 & 897 & 9.19 & $\cdots$ \\
\hline J2323.4+5849 & $23: 23: 28.85$ & $+58: 49: 09.48$ & 1.49 & 40.07 & 62.4 & 39.1 & 26.4 & 1.6 & 2568 & 9.17 & snr \\
\hline $\mathrm{J} 1625.1-0021^{\dagger}$ & $16: 25: 07.06$ & $-00: 21: 30.96$ & 3.38 & 37.31 & 104.3 & 201.4 & 1.9 & 0.8 & 1778 & 8.98 & $\cdots$ \\
\hline $\mathrm{J} 1714.5-3832$ & $17: 14: 34.27$ & $-38: 32: 55.68$ & 2.65 & 68.77 & 39.3 & 23.3 & 14.7 & 2.2 & 2649 & 8.95 & snr \\
\hline $\mathrm{J} 1857.9+0210^{\dagger}$ & $18: 57: 57.65$ & $+02: 10: 13.44$ & 5.41 & 50.62 & 42.8 & 50.5 & 3.2 & 1.9 & 601 & 8.89 & $\cdots$ \\
\hline J1056.7-5853 & $10: 56: 42.86$ & $-58: 53: 45.60$ & 7.77 & 35.71 & 88.2 & 126.1 & 1.1 & 1.5 & 596 & 8.83 & $\ldots$ \\
\hline $\mathrm{J} 1026.2-5730$ & $10: 26: 14.33$ & $-57: 30: 59.76$ & 4.85 & 50.42 & 54.7 & 58.1 & 2.3 & 1.6 & 493 & 8.26 & $\cdots$ \\
\hline J1742.6-3321 & $17: 42: 39.60$ & $-33: 21: 22.32$ & 6.00 & 48.24 & 67.1 & 24.4 & 2.5 & 1.8 & 411 & 8.20 & $\cdots$ \\
\hline $\mathbf{J 1 8 4 4 . 3}-0344^{\dagger}$ & $18: 44: 23.93$ & $-03: 44: 48.48$ & 5.09 & 44.78 & 37.0 & 70.9 & 1.9 & 0.8 & 468 & 8.12 & $\cdots$ \\
\hline J1101.9-6053 & 11:01:55.46 & $-60: 53: 45.96$ & 7.49 & 23.32 & 40.8 & 61.3 & 2.4 & 1.8 & 519 & 7.95 & spp \\
\hline J2038.4+4212 & $20: 38: 29.95$ & $+42: 12: 30.60$ & 5.30 & 45.67 & 51.1 & 95.8 & 0.5 & 0.6 & 340 & 7.92 & $\cdots$ \\
\hline J1849.4-0057 & $18: 49: 25.30$ & $-00: 57: 06.48$ & 3.55 & 45.11 & 23.8 & 16.6 & 13.5 & 2.0 & 674 & 7.86 & snr \\
\hline $\mathrm{J} 1112.0-6135$ & $11: 12: 04.03$ & $-61: 35: 03.12$ & 8.87 & 55.72 & 84.6 & 35.8 & 1.7 & 1.7 & 293 & 7.84 & $\cdots$ \\
\hline J1754.0-2538 & $17: 54: 02.02$ & $-25: 38: 54.96$ & 2.62 & 66.89 & 72.4 & 107.3 & 4.0 & 1.0 & 500 & 7.73 & $\cdots$ \\
\hline $\mathrm{J} 0854.8-4503^{\dagger}$ & 08:54:50.59 & $-45: 03: 41.76$ & 4.37 & 44.94 & 47.5 & 54.9 & 5.0 & 1.7 & 737 & 7.68 & $\ldots$ \\
\hline $\mathrm{J} 1857.2+0059$ & $18: 57: 14.28$ & $+00: 59: 10.68$ & 3.82 & 57.14 & 32.6 & 113.2 & 4.5 & 1.3 & 383 & 7.67 & $\cdots$ \\
\hline $\mathrm{J} 1740.5-2843$ & $17: 40: 30.00$ & $-28: 43: 01.20$ & 5.87 & 46.42 & 25.6 & 24.2 & 3.6 & 2.2 & 700 & 7.66 & $\cdots$ \\
\hline $\mathbf{J 1 7 4 4 . 1 - 7 6 1 9}$ & $17: 44: 10.85$ & $-76: 19: 42.96$ & 3.12 & 51.73 & 112.5 & 169.2 & 2.1 & 1.2 & 1759 & 7.61 & $\cdots$ \\
\hline $\mathbf{J} 1035.7-6720^{\dagger}$ & $10: 35: 42.24$ & $-67: 20: 00.60$ & 3.34 & 47.01 & 80.6 & 120.2 & 2.3 & 1.4 & 1336 & 7.39 & $\cdots$ \\
\hline $\mathrm{J} 1843.7-0322$ & $18: 43: 42.77$ & $-03: 22: 37.92$ & 7.67 & 70.63 & 65.5 & 54.5 & 3.7 & 2.6 & 1113 & 7.37 & $\cdots$ \\
\hline $\mathbf{J} 0359.5+5413^{\dagger}$ & 03:59:31.46 & $+54: 13: 19.20$ & 3.66 & 33.63 & 42.2 & 84.1 & 2.6 & 1.6 & 800 & 7.19 & $\cdots$ \\
\hline J1624.2-4041 ${ }^{\dagger}$ & $16: 24: 14.26$ & $-40: 41: 11.40$ & 4.02 & 50.80 & 58.8 & 74.2 & 2.8 & 1.6 & 945 & 7.18 & $\ldots$ \\
\hline $\mathrm{J} 1740.5-2726$ & $17: 40: 32.28$ & $-27: 27: 00.00$ & 8.30 & 43.15 & 39.9 & 31.1 & 1.8 & 2.0 & 401 & 7.04 & $\cdots$ \\
\hline J1827.3-1446 & $18: 27: 20.16$ & $-14: 46: 01.92$ & 5.54 & 40.00 & 18.2 & 83.5 & 2.5 & 1.4 & 483 & 6.96 & $\cdots$ \\
\hline $\mathrm{J} 2032.5+3921$ & $20: 32: 29.78$ & $+39: 25: 20.60$ & 3.69 & 49.41 & 46.2 & 34.1 & 0.4 & 0.8 & 233 & 6.95 & $\cdots$ \\
\hline J1638.6-4654 & $16: 38: 40.16$ & $-46: 54: 06.33$ & 2.24 & 77.58 & 48.0 & 46.8 & 3.7 & 1.8 & 614 & 6.84 & spp \\
\hline J1925.4+1727 & $19: 24: 58.98$ & $+17: 24: 41.84$ & 7.38 & 47.33 & 42.2 & 22.3 & 1.2 & 1.2 & 157 & 6.70 & $\ldots$ \\
\hline $\mathrm{J} 1857.9+0355$ & 18:58:03.73 & $+03: 55: 08.04$ & 3.45 & 55.58 & 31.5 & 29.6 & 1.6 & 1.1 & 146 & 6.55 & $\ldots$ \\
\hline J1208.4-6239 ${ }^{\dagger}$ & $12: 08: 26.89$ & $-62: 39: 26.13$ & 1.56 & 64.44 & 39.2 & 52.0 & 4.9 & 1.8 & 874 & 6.43 & $\cdots$ \\
\hline $\mathbf{J 1 3 5 0 . 4}-6224^{\dagger}$ & $13: 50: 34.69$ & $-62: 23: 43.53$ & 1.71 & 58.24 & 41.3 & 90.8 & 2.4 & 0.7 & 357 & 6.41 & $\cdots$ \\
\hline J1037.9-5843* & 10:38:01.49 & $-58: 44: 20.62$ & 4.29 & 38.88 & 24.9 & 163.9 & 0.4 & 0.0 & 391 & 6.32 & $\cdots$ \\
\hline $\mathrm{J} 2112.5-3044^{\dagger}$ & $21: 12: 32.39$ & $-30: 43: 58.53$ & 1.39 & 51.84 & 69.0 & 151.0 & 2.8 & 1.1 & 1805 & 6.25 & $\cdots$ \\
\hline $\mathrm{J} 1636.2-4709 \mathrm{c}$ & $16: 36: 22.32$ & $-47: 09: 53.05$ & 4.41 & 57.44 & 13.7 & 4.4 & $\cdots$ & 2.3 & 541 & 6.17 & spp \\
\hline J1358.5-6025 & $13: 58: 24.20$ & $-60: 25: 30.56$ & 2.44 & 53.16 & 32.8 & 21.1 & 5.7 & 2.2 & 639 & 6.15 & $\cdots$ \\
\hline J1048.2-5928 & $10: 48: 40.66$ & $-59: 26: 03.43$ & 3.98 & 65.78 & 101.1 & 60.4 & 1.5 & 1.4 & 381 & 6.11 & $\cdots$ \\
\hline J2034.6+4302 & $20: 34: 58.42$ & $+43: 05: 08.99$ & 6.30 & 41.40 & 50.7 & 112.7 & 0.4 & 0.3 & 324 & 6.11 & $\cdots$ \\
\hline $\mathrm{J} 1754.0-2930^{\dagger}$ & $17: 54: 14.33$ & $-29: 32: 08.04$ & 3.72 & 59.67 & 49.8 & 38.4 & 2.2 & 2.0 & 498 & 6.06 & $\ldots$ \\
\hline $\mathrm{J} 1214.0-6236^{\dagger}$ & $12: 14: 10.04$ & $-62: 36: 16.69$ & 1.98 & 58.02 & 20.3 & 15.7 & 13.1 & 2.2 & 789 & 6.05 & spp \\
\hline $\mathrm{J} 1652.8-4351$ & $16: 52: 32.63$ & $-43: 56: 50.10$ & 6.40 & 64.55 & 31.0 & 62.0 & 1.3 & 0.9 & 184 & 6.00 & $\cdots$ \\
\hline J1317.6-6315 & $13: 17: 35.62$ & $-63: 17: 18.00$ & 2.96 & 50.53 & 25.0 & 37.0 & 2.7 & 1.7 & 347 & 5.99 & $\cdots$ \\
\hline J2039.4+4111 & $20: 39: 45.84$ & $+41: 09: 34.39$ & 3.61 & 45.39 & 48.2 & 98.1 & 0.3 & 0.3 & 249 & 5.91 & $\cdots$ \\
\hline $\mathrm{J} 1852.8+0158^{*}$ & $18: 52: 27.92$ & $+02: 01: 37.54$ & 4.17 & 54.52 & 12.1 & 0.2 & $\cdots$ & 2.8 & 838 & 5.89 & $\cdots$ \\
\hline J0631.6+0644 & $06: 31: 49.76$ & $+06: 44: 46.66$ & 1.93 & 43.04 & 26.6 & 37.2 & 4.6 & 1.6 & 676 & 5.84 & spp \\
\hline $\mathrm{J} 1840.1-0412^{*}$ & $18: 40: 06.15$ & $-04: 11: 35.22$ & 2.95 & 30.14 & 15.9 & 0.0 & $\cdots$ & 2.5 & 416 & 5.83 & spp \\
\hline J1928.9+1739 & $19: 29: 02.93$ & $+17: 34: 58.90$ & 9.16 & 47.86 & 26.9 & 12.0 & 3.6 & 2.1 & 235 & 5.79 & $\cdots$ \\
\hline J0225.8+6159 & $02: 26: 20.37$ & $+62: 00: 10.48$ & 3.49 & 46.69 & 28.8 & 29.7 & 2.2 & 1.7 & 473 & 5.77 & $\ldots$ \\
\hline $\mathbf{J} 0002.6+6218^{\dagger}$ & 00:02:48.88 & $+62: 16: 54.71$ & 2.25 & 48.02 & 58.0 & 80.3 & 1.8 & 1.5 & 716 & 5.76 & $\cdots$ \\
\hline J1740.5-2642 & $17: 40: 41.52$ & $-26: 39: 52.98$ & 4.29 & 33.42 & 23.2 & 34.1 & 2.5 & 1.8 & 222 & 5.74 & $\cdots$ \\
\hline
\end{tabular}


Table 1

(Continued)

\begin{tabular}{|c|c|c|c|c|c|c|c|c|c|c|c|}
\hline 3FGL Name & $\begin{array}{l}\text { Searched R.A. } \\
\quad(\mathrm{J} 2000)\end{array}$ & $\begin{array}{l}\text { Searched Decl. } \\
\quad(\mathrm{J} 2000)\end{array}$ & $\begin{array}{l}\text { Search Radius } \\
\quad(\operatorname{arcmin})\end{array}$ & VI & $\mathrm{TS}_{\text {curve }}$ & $\mathrm{TS}_{\text {cut }}$ & $\begin{array}{l}E_{\mathrm{cut}} \\
(\mathrm{GeV})\end{array}$ & $\Gamma$ & TS & $\log R_{S}$ & Class \\
\hline $\mathrm{J} 1834.5-0841^{*}$ & $18: 34: 31.66$ & $-08: 40: 15.75$ & 4.02 & 57.10 & 0.5 & 0.1 & $\ldots$ & 2.2 & 287 & 5.72 & $\overline{\mathrm{snr}}$ \\
\hline J2042.4+4209 & $20: 42: 39.77$ & $+42: 09: 19.64$ & 11.48 & 49.90 & 27.1 & 27.4 & 0.5 & 1.0 & 185 & 5.68 & $\ldots$ \\
\hline $\mathrm{J} 1814.0-1757 \mathrm{c}$ & $18: 13: 24.52$ & $-17: 53: 55.97$ & 5.83 & 56.91 & 8.8 & 7.4 & $\ldots$ & 2.3 & 662 & 5.59 & $\ldots$ \\
\hline $\mathrm{J} 2041.1+4736^{\dagger}$ & $20: 41: 08.34$ & $+47: 35: 50.81$ & 2.01 & 56.28 & 38.0 & 15.9 & 10.3 & 2.3 & 967 & 5.53 & $\ldots$ \\
\hline J1047.3-6005 & $10: 47: 21.66$ & $-60: 05: 11.01$ & 6.22 & 49.04 & 22.3 & 16.4 & 3.0 & 1.5 & 115 & 5.52 & $\ldots$ \\
\hline J2039.6-5618 & $20: 39: 36.25$ & $-56: 17: 12.94$ & 1.82 & 34.60 & 30.4 & 60.3 & 3.9 & 1.6 & 1266 & 5.47 & $\ldots$ \\
\hline $\mathrm{J} 1900.8+0337$ & 19:00:37.96 & $+03: 39: 10.57$ & 3.94 & 45.87 & 44.9 & 4.7 & $\ldots$ & 2.3 & 186 & 5.42 & $\ldots$ \\
\hline J0855.4-4818 & $08: 55: 18.44$ & $-48: 14: 13.02$ & 10.69 & 33.84 & 53.0 & 66.4 & 0.5 & 0.9 & 288 & 5.39 & $\ldots$ \\
\hline J1747.7-2904 & $17: 47: 51.94$ & $-29: 01: 49.54$ & 2.95 & 65.34 & 10.3 & 124.7 & 7.1 & 2.2 & 666 & 5.37 & $\ldots$ \\
\hline $\mathrm{J} 0541.1+3553$ & $05: 40: 47.47$ & $+35: 54: 40.72$ & 8.53 & 35.17 & 37.3 & 33.0 & 1.8 & 1.9 & 329 & 5.34 & $\ldots$ \\
\hline $\mathrm{J} 1549.1-5347 \mathrm{c}^{*}$ & $15: 48: 38.12$ & $-53: 44: 00.33$ & 5.02 & 51.64 & 10.9 & 0.1 & $\ldots$ & 2.9 & 1172 & 5.27 & spp \\
\hline J1039.1-5809 & $10: 38: 25.85$ & $-58: 08: 23.45$ & 13.63 & 37.46 & 24.7 & 23.4 & 1.7 & 1.3 & 107 & 5.23 & \\
\hline J1831.7-0230 & $18: 31: 33.96$ & $-02: 31: 25.54$ & 5.83 & 31.11 & 17.8 & 2.1 & $\ldots$ & 2.7 & 421 & 5.23 & $\ldots$ \\
\hline $\mathrm{J} 1702.8-5656^{\dagger}$ & 17:02:45.00 & $-56: 54: 39.46$ & 1.88 & 58.78 & 46.9 & 53.1 & 3.4 & 2.1 & 1917 & 5.19 & $\ldots$ \\
\hline J1736.0-2701* & $17: 36: 07.44$ & $-27: 03: 29.55$ & 6.88 & 38.45 & 25.2 & 23.7 & 0.3 & 0.0 & 80 & 5.18 & $\ldots$ \\
\hline $\mathrm{J} 2023.5+4126^{*}$ & $20: 23: 24.65$ & $+41: 27: 31.08$ & 4.35 & 48.95 & 78.1 & 36.7 & 0.4 & 0.0 & 93 & 5.12 & $\ldots$ \\
\hline J1758.8-2346 & 17:59:09.58 & $-23: 47: 19.28$ & 3.69 & 41.80 & 11.8 & 5.4 & $\ldots$ & 1.9 & 218 & 5.01 & $\ldots$ \\
\hline J2004.4+3338* & $20: 04: 22.03$ & $+33: 39: 29.46$ & 1.47 & 50.29 & 13.5 & 0.0 & $\ldots$ & 2.4 & 708 & 5.01 & $\ldots$ \\
\hline $\mathrm{J} 0212.1+5320$ & $02: 12: 12.29$ & $+53: 20: 49.61$ & 1.58 & 51.47 & 45.9 & 82.0 & 3.3 & 1.5 & 1442 & 5.01 & $\ldots$ \\
\hline $\mathrm{J} 1901.1+0728$ & 19:01:09.32 & $+07: 30: 01.23$ & 3.29 & 55.34 & 25.8 & 10.5 & 6.6 & 2.0 & 134 & 4.88 & $\ldots$ \\
\hline J1503.5-5801 & 15:03:39.92 & $-58: 00: 43.22$ & 3.88 & 67.48 & 26.3 & 18.7 & 3.7 & 2.0 & 359 & 4.85 & $\ldots$ \\
\hline J1850.5-0024 & $18: 50: 31.56$ & $-00: 24: 33.69$ & 4.83 & 64.27 & 14.6 & 2.8 & $\ldots$ & 2.3 & 216 & 4.76 & $\ldots$ \\
\hline J0933.9-6232 $2^{\dagger}$ & 09:34:00.41 & $-62: 32: 57.43$ & 1.77 & 59.20 & 88.0 & 125.9 & 2.0 & 0.8 & 907 & 4.73 & $\ldots$ \\
\hline $\mathrm{J} 1620.0-5101$ & $16: 19: 48.66$ & $-51: 00: 57.34$ & 4.03 & 50.48 & 9.7 & 1.0 & $\ldots$ & 2.1 & 121 & 4.72 & $\ldots$ \\
\hline $\mathrm{J} 1726.6-3530 \mathrm{c}$ & $17: 26: 32.27$ & $-35: 33: 37.61$ & 5.18 & 60.31 & 11.9 & 1.8 & $\ldots$ & 2.6 & 335 & 4.67 & $\ldots$ \\
\hline J1919.9+1407 & $19: 20: 11.19$ & $+14: 11: 54.53$ & 7.95 & 67.73 & 17.6 & 0.3 & $\ldots$ & 2.7 & 642 & 4.66 & $\ldots$ \\
\hline $\mathrm{J} 1119.9-2204^{\dagger}$ & $11: 19: 59.45$ & $-22: 04: 25.17$ & 1.80 & 62.62 & 103.2 & 156.9 & 1.7 & 1.3 & 1949 & 4.63 & $\ldots$ \\
\hline J0907.0-4802* & 09:07:18.05 & $-47: 58: 38.32$ & 10.11 & 40.75 & 29.3 & 28.0 & 0.4 & 0.2 & 123 & 4.58 & $\ldots$ \\
\hline J1718.0-3726 & $17: 18: 02.10$ & $-37: 26: 50.06$ & 1.02 & 41.58 & 1.5 & 2.0 & $\ldots$ & 2.1 & 593 & 4.55 & snr \\
\hline J1859.6+0102 & $18: 59: 39.72$ & $+01: 00: 15.56$ & 5.43 & 68.61 & 18.9 & 13.1 & 3.5 & 1.8 & 150 & 4.40 & $\ldots$ \\
\hline $\mathrm{J} 2035.0+3634$ & 20:35:02.11 & $+36: 32: 12.74$ & 1.88 & 52.58 & 39.2 & 57.5 & 2.8 & 0.8 & 401 & 4.39 & $\ldots$ \\
\hline J1345.1-6224 & 13:44:43.61 & $-62: 28: 30.64$ & 5.12 & 58.30 & 12.8 & 1.3 & $\ldots$ & 2.7 & 568 & 4.39 & spp \\
\hline J0744.1-2523 & 07:44:06.64 & $-25: 25: 17.47$ & 1.97 & 61.34 & 40.9 & 55.3 & 3.2 & 1.8 & 666 & 4.27 & $\ldots$ \\
\hline J0426.7+5437 & 04:26:33.79 & $+54: 35: 00.35$ & 3.01 & 51.83 & 63.9 & 59.0 & 1.7 & 2.1 & 1235 & 4.27 & $\ldots$ \\
\hline $\mathrm{J} 1539.2-3324^{\dagger}$ & $15: 39: 20.23$ & $-33: 24: 56.62$ & 1.64 & 57.87 & 102.9 & 129.3 & 2.3 & 0.4 & 694 & 4.22 & $\ldots$ \\
\hline $\mathrm{J} 1641.1-4619 \mathrm{c}^{*}$ & $16: 41: 00.45$ & $-46: 19: 46.25$ & 1.87 & 39.43 & 0.7 & 0.2 & $\ldots$ & 2.3 & 292 & 4.15 & spp \\
\hline J1528.3-5836 & $15: 28: 23.37$ & $-58: 38: 05.98$ & 1.87 & 68.72 & 44.9 & 41.4 & 4.0 & 1.6 & 452 & 4.14 & \\
\hline J1857.9+0355 & $18: 58: 03.73$ & $+03: 55: 08.04$ & 3.45 & 41.47 & 11.2 & 32.2 & 2.2 & 1.4 & 131 & 4.13 & $\ldots$ \\
\hline $\mathrm{J} 1855.4+0454$ & $18: 55: 12.72$ & $+04: 55: 38.38$ & 4.46 & 38.60 & 6.6 & 4.4 & $\ldots$ & 2.4 & 193 & 4.12 & $\ldots$ \\
\hline $\mathrm{J} 1650.0-4438 \mathrm{c}^{*}$ & $16: 49: 48.42$ & $-44: 38: 58.44$ & 6.63 & 58.81 & 1.0 & 0.1 & $\ldots$ & 3.1 & 843 & 4.02 & $\ldots$ \\
\hline J0901.6-4700 & 09:01:40.90 & $-46: 52: 10.77$ & 7.02 & 55.10 & 30.0 & 52.7 & 1.0 & 1.2 & 221 & 4.02 & $\ldots$ \\
\hline J1329.8-6109 & $13: 29: 57.92$ & $-61: 08: 00.95$ & 2.45 & 55.66 & 22.5 & 21.1 & 4.9 & 1.6 & 246 & 3.91 & $\ldots$ \\
\hline J1639.4-5146 & $16: 39: 25.17$ & $-51: 46: 04.03$ & 1.39 & 58.03 & 4.2 & 2.8 & $\ldots$ & 2.3 & 945 & 3.85 & $\ldots$ \\
\hline J1833.9-0711* & $18: 34: 10.57$ & $-07: 11: 34.47$ & 3.12 & 82.07 & 1.6 & 0.4 & $\ldots$ & 2.3 & 482 & 3.85 & spp \\
\hline $\mathrm{J} 1814.1-1734 \mathrm{c}$ & 18:14:07.87 & $-17: 36: 39.99$ & 2.96 & 50.07 & 7.1 & 5.3 & $\ldots$ & 1.4 & 83 & 3.73 & \\
\hline J1139.0-6244 & 11:39:07.61 & $-62: 46: 04.02$ & 2.31 & 29.45 & 7.5 & 16.5 & 8.6 & 1.9 & 278 & 3.71 & \\
\hline $\mathrm{J} 1626.2-2428 \mathrm{c}$ & $16: 26: 25.40$ & $-24: 31: 36.54$ & 4.74 & 46.87 & 15.9 & 7.8 & $\ldots$ & 2.1 & 392 & 3.66 & 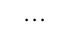 \\
\hline 3FGL J1212.2-6251 & $12: 12: 18.06$ & $-62: 53: 31.51$ & 2.84 & 53.70 & 1.4 & 12.9 & 45.8 & 2.4 & 426 & 3.45 & spp \\
\hline
\end{tabular}

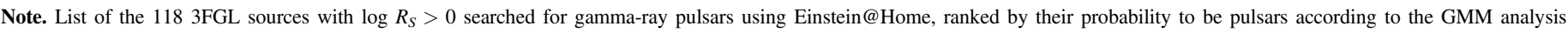

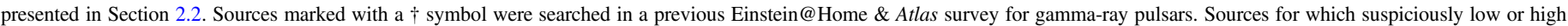

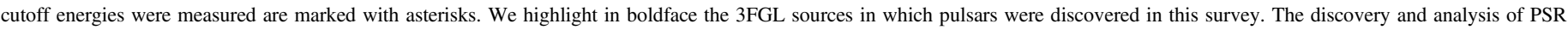

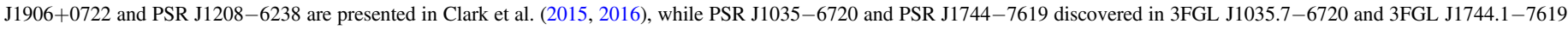

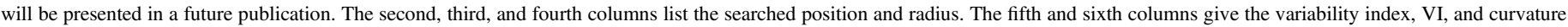

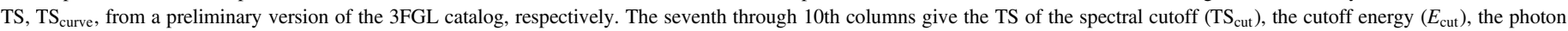

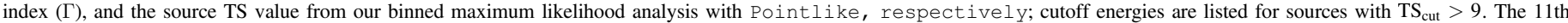

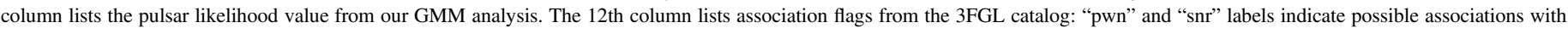

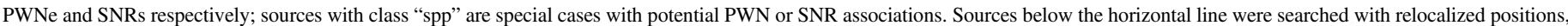
as mentioned in Section 3.2.

(This table is available in machine-readable form.)

$1 \sigma$ Gaussian uncertainty reported by Pointlike to obtain a more conservative sky coverage, and we also extended our data set by including photons recorded until 2015 July 7 when the relocalization was done. The inaccurate source locations might have resulted from the imperfect Galactic background model.

\subsection{Search Summary}

The blind search survey of the sources listed in the Appendix, described in detail in Paper I, enabled the discovery of 17 gamma-ray pulsars. Clark et al. (2015) reported on the discovery of PSR J1906+0722, an energetic pulsar with a spin frequency 

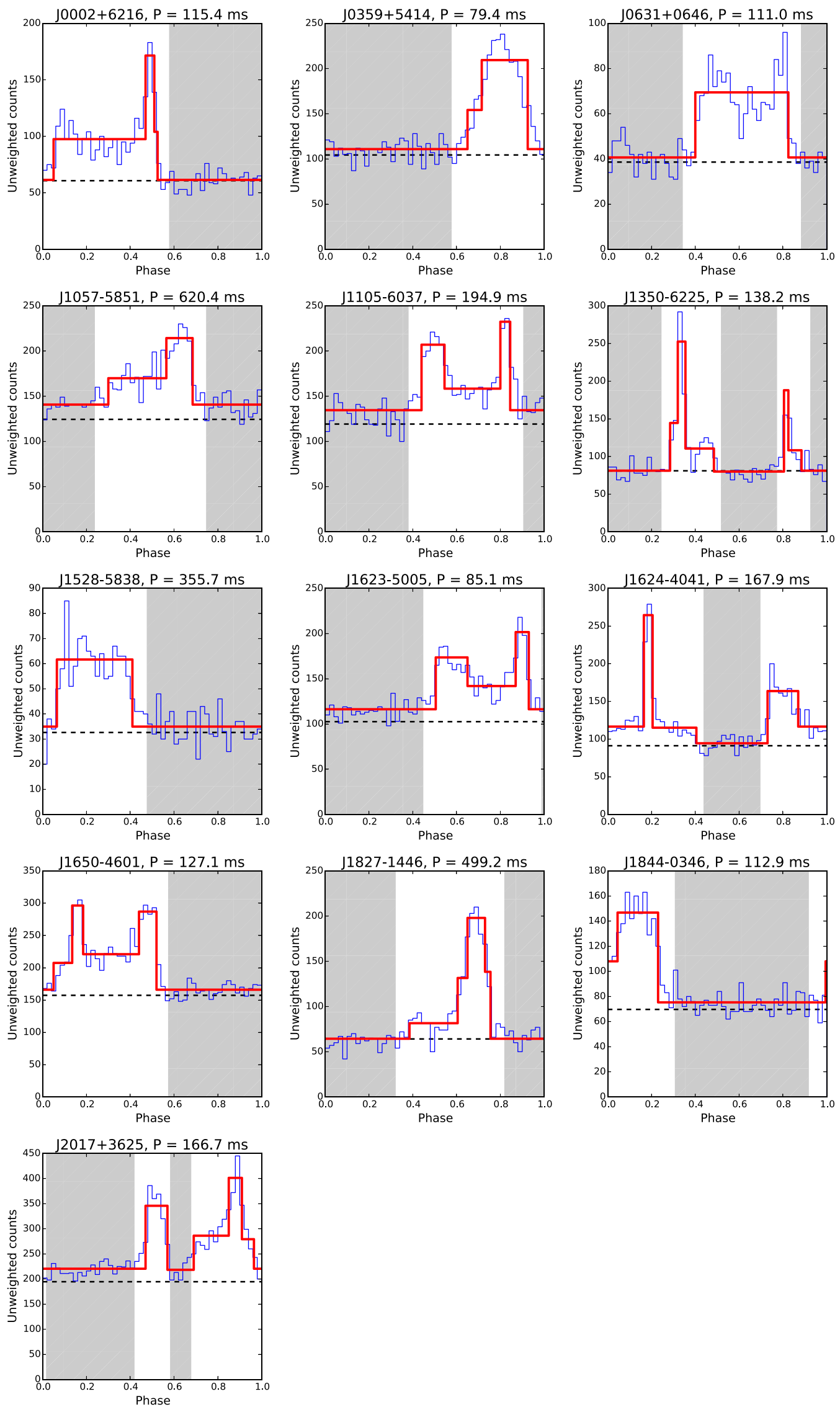

Figure 1. Results of the decomposition of gamma-ray pulse profiles into Bayesian blocks, as discussed in Section 4.1. Blue histograms represent the pulse profiles, red lines the Bayesian block decompositions, and shaded regions the off-pulse phase intervals determined from this analysis. Dashed black lines represent the estimated background levels, calculated as $B=\sum_{i}^{N}\left(1-w_{i}\right)$, where $w_{i}$ is the weight associated with photon $i$ (Guillemot et al. 2012b). 
of $8.9 \mathrm{~Hz}$ that suffered one of the largest glitches ever observed for a gamma-ray pulsar. Clark et al. (2016) later presented the discovery of PSR J1208-6238, a $2.3 \mathrm{~Hz}$ pulsar with a very high surface magnetic field and a measurable braking index of about 2.6. Paper I and the present paper report on 13 young, isolated gamma-ray pulsars also found in this survey. The new pulsars have rotational periods ranging from $\sim 79 \mathrm{~ms}$ to $620 \mathrm{~ms}$. They are all energetic, with spin-down powers between about $10^{34}$ and $4 \times 10^{36} \mathrm{erg} \mathrm{s}^{-1}$. Among these, PSR J1057 -5851 and PSR J1827-1446 are the slowest rotators among currently known gamma-ray pulsars. PSR J1844-0346 experienced a very large glitch in mid-2012 (see Paper I for details). In the next sections we describe dedicated follow-up studies of these 13 pulsars. Finally, we note that two more pulsars were found in this survey: PSR J1035-6720 and PSR J1744-7619. These two pulsars will be presented in a separate publication (Clark et al. 2017).

\section{Follow-up Analysis}

\subsection{Spectral Analysis}

After selecting the 13 pulsars, we performed dedicated spectral analyses with extended data sets in order to characterize their spectral properties with extra sensitivity. We used updated Pass 8 (P8R2) event selections and updated IRFs for events recorded from 2008 August 4 until 2015 September 9. The sizes of the ROIs around each pulsar were extended to $15^{\circ}$ to collect more gamma-ray photons for the follow-up analysis, and we selected photon energies $>100 \mathrm{MeV}$ and zenith angles $<90^{\circ}$. The more restrictive zenith angle cut was used to better reject events from the Earth's limb in support of spectral analysis down to $100 \mathrm{MeV}$. In our dedicated spectral analyses we used the gll_iem_v06.fit.s ${ }^{24}$ map cube and iso_P8R2_SOURCE_V6_v06.txt ${ }^{25}$ template for modeling the Galactic diffuse emission and the isotropic diffuse background, to match with the current recommendations (Acero et al. 2016a). The numbers of point sources in the models were increased to include all 3FGL sources within $20^{\circ}$. The details of the timing analysis using these extended data sets, including the determination of timing and positional parameters, are presented in Paper I. For the spectral analysis of the 13 pulsars we used the positions obtained from pulsar timing. In order to further minimize contamination from the diffuse background or from neighboring sources, we restricted our data sets to the pulsed part of the pulse profiles. To determine the "on"- and "off"-pulse phase regions of the pulse profiles, we selected gamma-ray photons with weights above 0.05 and constructed unweighted pulse profiles, which we then analyzed with the Bayesian block decomposition method described by Scargle et al. (2013). Bayesian blocks represent a model of time series of events generated by an inhomogeneous Poisson process, involving a sequence of constant flux levels. This method is useful for discriminating random flux changes from real ones, but it is not a physical representation of the pulse profiles. The on- and off-pulse regions are shown in Figure 1. We selected photons in the on-pulse regions and performed spectral analyses of these restricted data sets. We determined the

\footnotetext{
${ }^{24}$ https://fermi.gsfc.nasa.gov/ssc/data/analysis/software/aux/gll_iem_ v06.fits

${ }^{25}$ https://fermi.gsfc.nasa.gov/ssc/data/analysis/software/aux/iso_P8R2_ SOURCE_V6_v06.txt
}

significance of the spectral cutoff $\left(\mathrm{TS}_{\mathrm{cut}}\right)$ by comparing the change in log-likelihood when using a simple power-law model for the spectra of the pulsars instead of assuming the PLEC model, as follows: $\mathrm{TS}_{\text {cut }}=-2 \log \Delta \mathcal{L}$. The results of the spectral analysis of the on-pulse data are given in Table 3; the corresponding SEDs are displayed in Figure 2, and the best-fit cutoff energy and power-law index values are shown in Figure 3, along with those of 2PC pulsars.

To search for unpulsed magnetospheric pulsar emission or emission from a putative PWN associated with the pulsar, we conducted analyses of the off-pulse phases of the data sets. Point-like test sources were added to the spectral models at the locations of the pulsars, and the spectral properties of these sources were determined by running new likelihood analyses. We alternatively assumed a simple power-law model and a PLEC model for the test sources, in order to test for spectral curvature. Significant off-pulse emission was detected for PSR J1623-5005, PSR J1624-4041, and PSR J2017+3625, with evidence for spectral curvature suggestive of magnetospheric emission from the pulsars, as can be seen from Table 4. Such off-pulse pulsar emission is not atypical for known gamma-ray pulsars (see, e.g., 2PC); nevertheless, small, unmodeled spatial fluctuations in the bright diffuse background emission could also account for this emission. Detailed analyses with extended data sets and comparisons of the best-fit spectral parameters with those of other known gamma-ray pulsars with off-pulse emission are necessary to firmly establish PSR J1623-5005, PSR J1624-4041, and PSR J2017+3625 as pulsars exhibiting gamma-ray emission at all phases. The on-pulse emission was then refitted with the addition of sources detected in the offpulse region scaled to the on-pulse interval with the normalization and spectral parameters fixed.

We characterized the pulse profiles displayed in Figure 5 of Paper I by fitting the weighted profiles to Gaussian or Lorentzian profiles, depending on which gave a higher log likelihood. The derived peak multiplicities and gamma-ray peak separations are reported in Table 5. Most of the new pulsars show double-peaked profiles, with well-separated components that are typical of young gamma-ray pulsar light curves (see 2PC). Two of the 13 newly discovered pulsars, PSR J0002+6216 and PSR J0631+1036, are detected in the radio band (see Section 4.2). For these pulsars we measured the phase offset between the radio peak and the first gammaray peak.

\subsection{Radio Counterpart Searches}

The new pulsars were searched for radio pulsations by reanalyzing archival observations from previous targeted radio surveys of Fermi LAT unassociated sources, or by conducting new dedicated observations. Because we have timing parameters for the new pulsars, the only parameter to search for when analyzing the radio observations is the dispersion measure (DM), a quantity representing the integrated column density of free electrons along the line of sight to the pulsars, causing radio waves to arrive at different times depending on the frequency. Radio observations were therefore folded at the periods determined from the gamma-ray timing (see Paper I) and searched in DM values only, resulting in a reduced number of trials compared to a typical radio pulsar search.

The list of telescopes and backends used is given in Table 6. For each observing configuration we give the gain $G$, the 
Table 2

Relocalization Results

\begin{tabular}{lccccc}
\hline \hline PSR & 3FGL Source & $\begin{array}{c}r_{95} \\
(3 F G L)\end{array}$ & $\begin{array}{c}r_{95} \\
(\text { new })\end{array}$ & $\Delta_{3 \text { FGL }}$ & $\Delta_{\text {new }}$ \\
\hline J0002+6216 & J0002.6+6218 & $3 ! 6$ & $2 ! 0$ & $2 ! 7$ & $1 ! 3$ \\
J0359+5414 & J0359.5+5413 & $2 ! 4$ & $1 ! 8$ & $1 ! 8$ & $0 ! 6$ \\
J0631+0646 & J0631.6+0644 & $2 ! 8$ & $1 ! 8$ & $4 ! 1$ & $1 ! 6$ \\
J1057-5851 & J1056.7-5853 & $5 ! 2$ & $2 ! 5$ & $4 ! 4$ & $2 ! 9$ \\
J1105-6037 & J1104.9-6036 & $2 ! 7$ & $1 ! 5$ & $0 ! 7$ & $0 ! 9$ \\
J1350-6225 & J1350.4-6224 & $2 ! 4$ & $1 ! 6$ & $2 ! 0$ & $2 ! 3$ \\
J1528-5838 & J1528.3-5836 & $3 ! 3$ & $1 ! 7$ & $1 ! 7$ & $0 ! 1$ \\
J1623-5005 & J1622.9-5004 & $1 ! 5$ & $1 ! 9$ & $1 ! 7$ & $1 ! 5$ \\
J1624-4041 & J1624.2-4041 & $2 ! 7$ & $1 ! 6$ & $0 ! 9$ & $1 ! 4$ \\
J1650-4601 & J1650.3-4600 & $2 ! 1$ & $1 ! 3$ & $1 ! 9$ & $0 ! 9$ \\
J1827-1446 & J1827.3-1446 & $3 ! 7$ & $1 ! 6$ & $1 ! 2$ & $0 ! 7$ \\
J1844-0346 & J1844.3-0344 & $3 ! 4$ & $1 ! 6$ & $2 ! 8$ & $2 ! 3$ \\
J2017+3625 & J2017.9+3627 & $2 ! 1$ & $1 ! 2$ & $2 ! 4$ & $0 ! 9$ \\
\hline
\end{tabular}

Note. Results of the relocalization analysis discussed in Section 3.2. For each of the 13 new pulsars reported in Paper I, the second column lists the name of the 3FGL source in which the pulsar was discovered. The third and fourth columns list the semimajor axis of the 3FGL source error ellipse at $95 \%$ confidence $\left(r_{95}\right)$ and the semimajor axis value from our analysis, respectively. The $r_{95}$ (new) values are based on statistical uncertainties only. The fifth and sixth columns list the offset between pulsar timing positions and 3FGL positions $\left(\Delta_{3 \mathrm{FGL}}\right)$ and the offset between pulsar timing positions and new positions $\left(\Delta_{\text {new }}\right)$, respectively.

central frequency, the frequency bandwidth $\Delta F$, the sensitivity degradation factor $\beta$, the number of polarizations $n_{p}$, the halfwidth at half-maximum of the radio beam (HWHM), and the receiver temperature $T_{\text {rec }}$. Table 7 lists the radio observations processed in our follow-up study. Sensitivities were calculated using the modified radiometer equation given in Lorimer \& Kramer (2005):

$$
S_{\min }=\beta \frac{5 T_{\text {sys }}}{G \sqrt{n_{p} t_{\text {int }} \Delta F}} \sqrt{\frac{W}{P-W}},
$$

where a value of 5 is assumed for the threshold signal-to-noise ratio for a detection, $T_{\text {sys }}=T_{\text {rec }}+T_{\text {sky }}$, $t_{\text {int }}$ is the integration time, $P$ is the rotational period, and $W$ is the pulse width, assumed to be $0.1 \times P$. The quantity $T_{\text {sky }}$ is the temperature from the Galactic synchrotron component, estimated by scaling the $408 \mathrm{MHz}$ map of Haslam et al. (1982) to the observing frequency, assuming a spectral index of -2.6 . For some observations the pointing direction was offset from the actual sky location of the pulsar. In those cases the flux density limit $S_{\text {min }}$ as calculated using Equation (2) was divided by $e^{-(\theta / \mathrm{HWHM})^{2} / 1.5}$, where $\theta$ is the offset. For the majority of pulsars we failed to detect pulsations in the radio data and placed limits on their radio flux densities.

For two pulsars, PSR J0002+6216 and PSR J0631+0646, the analysis resulted in the detection of significant radio pulsations. PSR J0002+6216 was detected in a $2 \mathrm{hr}$ observation conducted at $1.4 \mathrm{GHz}$ with the Effelsberg radio telescope, with a DM of 218.6(6) $\mathrm{pc} \mathrm{cm}^{-3}$. PSR J0631+0636 was detected with Arecibo at $327 \mathrm{MHz}$ and at $1.4 \mathrm{GHz}$ in 70-minute observations and was also seen with Effelsberg at $1.4 \mathrm{GHz}$ during a $2 \mathrm{hr}$ follow-up observation. The best determined DM value from the Arecibo $327 \mathrm{MHz}$ observation was $195.2(2) \mathrm{pc} \mathrm{cm}^{-3}$. Phase-aligned radio and gamma-ray pulse profiles for PSR J0002+6216 and PSR J0631+0646 are displayed in Figure 4. In both cases the gamma-ray emission is seen to lag the weak radio emission, as commonly observed in other radio and gamma-ray pulsars, and suggesting that radio and gamma-ray emissions have different magnetospheric origins (see, e.g., 2PC).

\subsection{Pulse Profile Modeling}

Using photons selected within $5^{\circ}$ radius around the pulsars, we constructed weighted counts pulse profiles with 90,60 , or 30 bins if the weighted H-test TS, the statistical test for pulsation significance (de Jager et al. 1989), for a given pulsar was $\geqslant 1000$, between 100 and 1000 , or $<100$, respectively. For PSR J0002+6216 and PSR J0631+0646 with radio detections, we rebinned the radio pulse profiles to have the same number of bins as the corresponding gamma-ray profile. We performed likelihood fits, minimizing $-\ln \mathcal{L}$, where $\mathcal{L}$ is the likelihood value, of the gamma-ray pulse profile or the combination of the radio and gamma-ray pulse profiles, of all 13 pulsars using the geometric simulations and fitting technique of Johnson et al. (2014).

Following Clark et al. (2016), we used simulations with $P=100 \mathrm{~ms}$ and $\dot{P}=10^{-15} \mathrm{~s} \mathrm{~s}^{-1}$ and constructed likelihood values using a $\chi^{2}$ statistic. Each pulsar was fit using the outer gap (OG, e.g., Cheng et al. 1986) and the slot gap (e.g., Muslimov \& Harding 2003, 2004) models, where we used the two-pole caustic model (TPC; Dyks \& Rudak 2003) as a geometric representation of the slot gap. For both models we use the vacuum retarded dipole solution for the magnetic field geometry (Deutsch 1955). The simulations were produced with $1^{\circ}$ resolution in both the magnetic inclination angle $(\alpha)$ and observer angle $(\zeta)$ and a resolution of $1 \%$ of the polar cap opening angle in emitting and accelerating gap widths. For radio simulations, we assumed a frequency of $1400 \mathrm{MHz}$ with the conal geometry and emission altitude of Story et al. (2007).

The best-fit results for all but PSR J0631+0646 are given in Table 8; estimated uncertainties are quoted at the $95 \%$ confidence level, but note that systematic error estimates from the fitting method (see Johnson et al. 2014) and/or from fitting only the gamma-ray profiles (Pierbattista et al. 2015) could be as large as $10^{\circ}$. Johnson et al. (2014) noted that it was necessary to renormalize the $\Delta \ln \mathcal{L}$ surface, making the best fit approximately correspond to a reduced $\chi^{2}$ value of 1 , in order to have more realistic confidence contours. In some cases, however, we found that this renormalization was unnecessary, either having no effect on the estimated uncertainties or shrinking them. We denote the pulsars for which we did not renormalize the likelihood surface with $\mathrm{a} \dagger$ in the first column of Table 8. For each model, we also estimated the beaming fraction $f_{\Omega}$ (as defined, e.g., in Watters et al. 2009; Venter et al. 2009) for the best-fit geometry, used when calculating the gamma-ray luminosity.

For each pulsar with no radio detection, we examined the simulated radio sky map at $1400 \mathrm{MHz}$, and evaluated the model predictions for the best-fit geometry, in regard to expected radio-loudness; the predictions are indicated in the sixth and 11th columns of Table 8 . The model predictions are "L" for radio-loud, meaning that the predicted geometry has the radio cone clearly and strongly intersecting our line of sight; " $F$ " for radio-faint, meaning the predicted geometry has our line of sight either narrowly missing the cone or clipping the very edge, suggesting that only weak emission would be detected; 

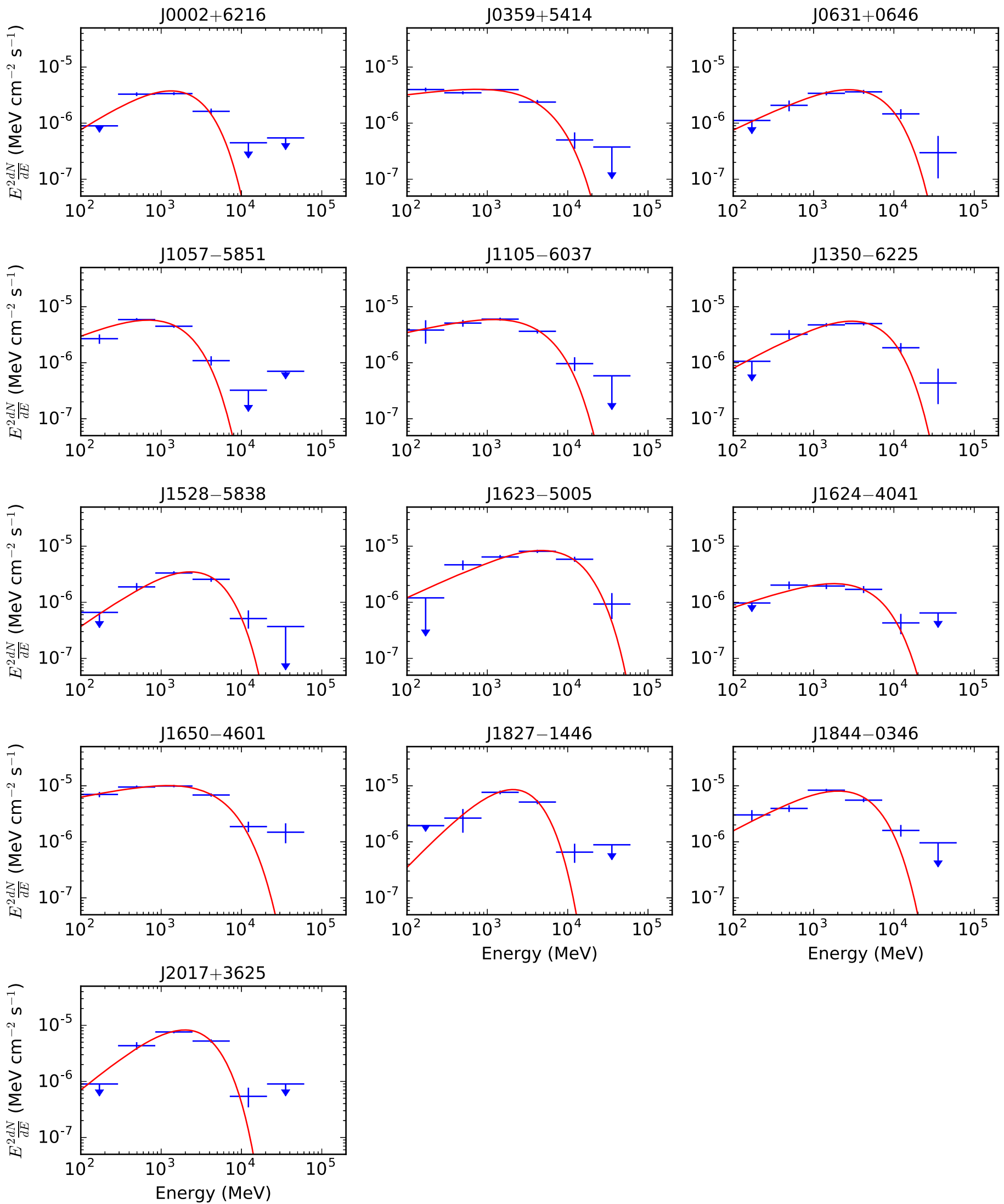

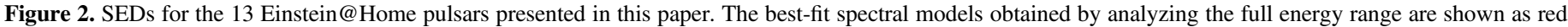
lines. The $95 \%$ confidence upper limits are calculated for energy bins with TS values below 4. 


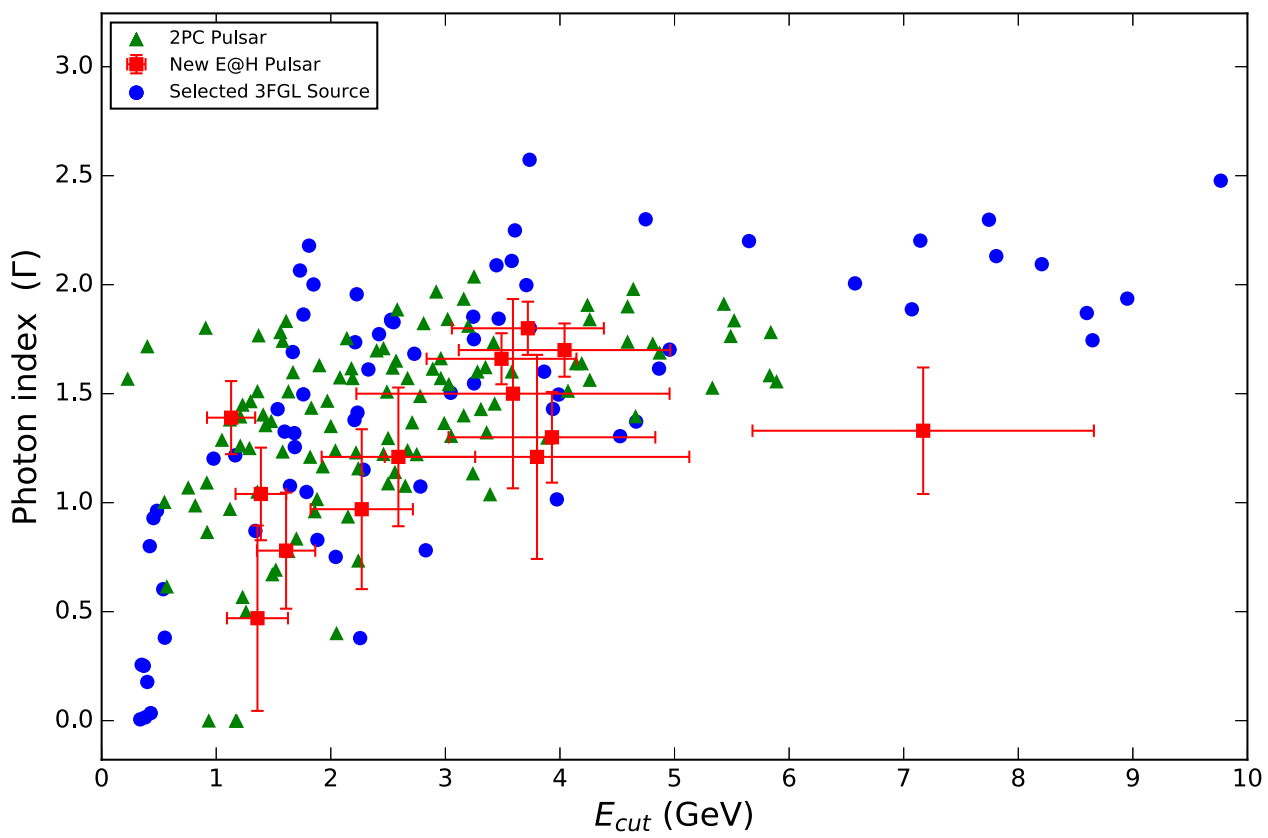

Figure 3. Best-fit power-law index $\Gamma$ vs. cutoff energy $E_{\text {cut }}$ for the new pulsars (red squares), other selected 3FGL sources that were searched in our survey (blue circles), and known gamma-ray pulsars from 2PC (green triangles). 3FGL sources with cutoff energies above $10 \mathrm{GeV}$ are not plotted and uncertainties are not displayed, to improve readability.

Table 3

On-pulse Spectral Parameters

\begin{tabular}{|c|c|c|c|c|c|c|}
\hline PSR & $\mathrm{TS}$ & $\mathrm{TS}_{\text {cut }}$ & $\Gamma$ & $\begin{array}{c}E_{\mathrm{cut}} \\
(\mathrm{GeV})\end{array}$ & $\begin{array}{l}\text { Photon Flux, } F_{100} \\
\left(10^{-8} \mathrm{ph} \mathrm{cm}^{-2} \mathrm{~s}^{-1}\right)\end{array}$ & $\begin{array}{c}\text { Energy Flux, } G_{100} \\
\left(10^{-11} \mathrm{erg} \mathrm{cm}^{-2} \mathrm{~s}^{-1}\right)\end{array}$ \\
\hline $\mathrm{J} 0002+6216$ & 975 & 145 & $1.04 \pm 0.16 \pm 0.14$ & $1.39 \pm 0.21 \pm 0.07$ & $2.8 \pm 0.3 \pm 0.8$ & $2.6 \pm 0.2 \pm 0.4$ \\
\hline $\mathrm{J} 0359+5414$ & 1610 & 93 & $1.80 \pm 0.07 \pm 0.10$ & $3.72 \pm 0.61 \pm 0.26$ & $8.4 \pm 0.6 \pm 2.0$ & $5.6 \pm 0.2 \pm 0.8$ \\
\hline $\mathrm{J} 0631+0646$ & 881 & 81 & $1.30 \pm 0.17 \pm 0.12$ & $3.93 \pm 0.84 \pm 0.33$ & $2.9 \pm 0.6 \pm 0.6$ & $3.7 \pm 0.3 \pm 0.3$ \\
\hline $\mathrm{J} 1057-5851$ & 813 & 123 & $1.39 \pm 0.16 \pm 0.05$ & $1.13 \pm 0.19 \pm 0.09$ & $7.9 \pm 0.9 \pm 0.8$ & $5.0 \pm 0.3 \pm 0.5$ \\
\hline $\mathrm{J} 1105-6037$ & 1084 & 94 & $1.66 \pm 0.11 \pm 0.04$ & $3.49 \pm 0.60 \pm 0.26$ & $8.3 \pm 1.2 \pm 0.4$ & $6.4 \pm 0.5 \pm 0.4$ \\
\hline $\mathrm{J} 1350-6225$ & 704 & 85 & $1.21 \pm 0.16 \pm 0.44$ & $3.80 \pm 0.70 \pm 1.13$ & $4.2 \pm 0.8 \pm 4.1$ & $6.0 \pm 0.4 \pm 2.1$ \\
\hline $\mathrm{J} 1528-5838$ & 593 & 87 & $0.97 \pm 0.07 \pm 0.36$ & $2.27 \pm 0.12 \pm 0.43$ & $2.2 \pm 0.5 \pm 1.3$ & $3.0 \pm 0.3 \pm 0.7$ \\
\hline $\mathrm{J} 1623-5005$ & 854 & 106 & $1.33 \pm 0.01 \pm 0.29$ & $7.17 \pm 0.17 \pm 1.48$ & $4.7 \pm 0.1 \pm 3.3$ & $8.1 \pm 0.2 \pm 2.0$ \\
\hline J1624-4041 & 255 & 31 & $1.50 \pm 0.21 \pm 0.38$ & $3.59 \pm 1.07 \pm 0.85$ & $1.6 \pm 0.8 \pm 0.9$ & $1.6 \pm 0.5 \pm 0.5$ \\
\hline $\mathrm{J} 1650-4601$ & 1368 & 83 & $1.70 \pm 0.10 \pm 0.07$ & $4.04 \pm 0.71 \pm 0.59$ & $15.9 \pm 1.4 \pm 4.6$ & $12.3 \pm 0.6 \pm 2.3$ \\
\hline $\mathrm{J} 1827-1446$ & 818 & 134 & $0.47 \pm 0.28 \pm 0.32$ & $1.36 \pm 0.22 \pm 0.15$ & $3.7 \pm 0.7 \pm 1.0$ & $5.8 \pm 0.5 \pm 0.6$ \\
\hline $\mathrm{J} 1844-0346$ & 840 & 75 & $1.21 \pm 0.22 \pm 0.23$ & $2.59 \pm 0.53 \pm 0.41$ & $8.3 \pm 1.9 \pm 2.6$ & $9.5 \pm 0.9 \pm 1.5$ \\
\hline $\mathrm{J} 2017+3625$ & 1148 & 216 & $0.78 \pm 0.15 \pm 0.22$ & $1.61 \pm 0.18 \pm 0.18$ & $4.7 \pm 1.3 \pm 1.3$ & $6.2 \pm 1.1 \pm 1.2$ \\
\hline
\end{tabular}

Note. Binned maximum likelihood spectral fit results for the 13 Einstein@Home gamma-ray pulsars. For each pulsar, the second and third columns list the TS of the source and the cutoff TS for the exponentially cutoff model over a simple power-law model, respectively. The fourth and fifth columns list the best-fit photon index $\Gamma$ and cutoff energy $E_{\text {cut }}$, respectively. The next two columns give the on-pulse phase-averaged integral photon and energy fluxes in the $0.1-100 \mathrm{GeV}$ band, $F_{100}$ and $G_{100}$, scaled to full interval values. The first reported uncertainties are statistical, while the second uncertainties are systematic, determined by reanalyzing the data with bracketing IRFs and artificially changing the normalization of the Galactic diffuse model by $\pm 6 \%$, as described in Acero et al. (2016b).

and "Q" for radio-quiet, meaning that the predicted geometry has our line of sight clearly missing the radio cone. The radiofaint sources are of particular interest, as searches at frequencies lower than $1400 \mathrm{MHz}$, where the cone is predicted to be larger (e.g., Story et al. 2007), may yield detections. Following Johnson et al. (2014), we conservatively consider one model to be significantly favored over another, for a given pulsar, if the $\ln \mathcal{L}$ value is greater by at least 15 ; however, in some cases the best-fit geometry for the TPC model clearly predicts a radio-loud pulsar where none has been detected, and we therefore claim that the OG model is favored, regardless of the $\Delta \ln \mathcal{L}$ value. In particular, this is the case for PSR J0359 +5414 , PSR J1528-5838, and PSR J1827-1446. For PSR
J1350-6225, both the TPC and OG models predict a radioloud pulsar, with a near-orthogonal rotator viewed near the spin equator, either casting doubts on the models or raising questions concerning the nondetection. In modeling the "radio-quiet" pulsars in 2PC, Pierbattista et al. (2015) similarly found some solutions where the line of sight was near enough to the magnetic axis that we might expect to intersect the radio emission cone. These authors used a different fitting technique but similar simulations. This may further suggest that our results regarding the aforementioned pulsars point to issues with the models and not with the nondections in radio.

Our joint gamma-ray and radio fits of PSR J0631+0646 did not produce acceptable results: the standard approach tended to 
Table 4

Off-pulse Spectral Parameters

\begin{tabular}{lrccc}
\hline \hline PSR & TS & $\mathrm{TS}_{\text {cut }}$ & $\Gamma$ & $\begin{array}{c}E_{\text {cut }} \\
(\mathrm{GeV})\end{array}$ \\
\hline $\mathrm{J} 1623-5005$ & 57 & 18 & $\ldots^{\mathrm{a}}$ & $0.87 \pm 0.07 \pm 0.21$ \\
$\mathrm{~J} 1624-4041$ & 47 & 10 & $1.02 \pm 0.95 \pm 0.96$ & $1.33 \pm 1.23 \pm 0.41$ \\
$\mathrm{~J} 2017+3625$ & 215 & 88 & $0.69 \pm 0.06 \pm 0.06$ & $0.59 \pm 0.01 \pm 0.06$
\end{tabular}

Note. Results of the maximum likelihood analysis of the off-pulse phase ranges of pulsars with significant off-pulse emission, as discussed in Section 4.1. The first column lists the name of the pulsar. The remaining columns list the TS of the source in the off-pulse phase range, the test statistic $\mathrm{TS}_{\text {cut }}$ of an exponentially cutoff model over a simple power-law model, the photon index $\Gamma$, and the energy cutoff $E_{\text {cut }}$.

${ }^{\text {a }}$ Although the spectral index is consistent with zero, the well-defined $E_{\text {cut }}$ allows integration to a finite total flux.

ignore the radio data. Following Johnson et al. (2014), we decreased the radio uncertainty value in order to increase its importance in the likelihood, but this proved ineffective, leading to fits that ignored the gamma-ray data. Under the assumption that the difficulty was in matching the observed phase lag between the radio peak and the gamma-ray peaks, we followed Guillemot et al. (2013) in allowing the phase of the magnetic pole in the radio and gamma-ray simulations to be different by as much as 0.1 (following realistic simulations of the pulsar magnetosphere by Kalapotharakos et al. (2012), which suggested an offset of the low-altitude polar gap from the outer magnetosphere by up to this amount). These new fits were, similarly, unsatisfactory. We investigated relaxing the maximum phase offset condition and found more acceptable fits with offsets of $\sim 0.3$ in phase for both the TPC and OG models. The maximum phase offset of 0.1 is inferred from Kalapotharakos et al. (2012) by comparing predicted light curves from the vacuum retarded dipole geometry to models with increasing conductivity and finally full force-free models. It seems implausible that this offset could be a factor of 3 larger than predicted in the force-free simulations. With our different attempts to model the radio and gamma-ray profiles jointly being unsuccessful, we do not report modeling results for PSR J0631+0646 in Table 8. New approaches for modeling this pulsar's emission geometry are needed. For instance, based on the work of Kalapotharakos et al. (2014), it is possible that gamma-ray emission from the current sheet outside the light cylinder could explain the extra phase lag for PSR J0631 +0646 , as their simulations did tend to show larger radio to gamma-ray phase lags.

\subsection{Luminosity, Distance, and Gamma-Ray Efficiency}

The fraction of their energy budgets that pulsars convert into gamma-ray radiation is a key question for understanding pulsar emission mechanisms. This requires converting the measured energy flux in gamma rays $G_{100}$ (see Table 3 for the values) into the gamma-ray luminosity, with the relation $L_{\gamma}=$ $4 \pi f_{\Omega} G_{100} d^{2}$, where $d$ is the distance. As discussed in Section 4.2, most of the 13 Einstein@Home pulsars considered in this study are undetected in radio. For these pulsars we therefore cannot use the DM to infer distances, e.g., using the NE2001 model of free electrons in the Galaxy (Cordes \& Lazio 2002). We can, however, calculate "heuristic" distances, $d_{h}$, and luminosities, $L_{\gamma}^{h}$, as follows:

$$
d_{h}=\sqrt{L_{\gamma}^{h} / 4 \pi G_{100}}
$$

where

$$
L_{\gamma}^{h}=\sqrt{\dot{E} / 10^{33} \mathrm{erg} \mathrm{s}^{-1}} \times 10^{33} \mathrm{erg} \mathrm{s}^{-1},
$$

i.e., assuming that the gamma-ray luminosity scales as $\sqrt{\dot{E}}$ for these young pulsars (see 2PC) and assuming a typical geometrical factor $f_{\Omega}$ of 1 . Heuristic distances for the 13 pulsars are given in Table 5. In most cases the values suggest that the pulsars lie at small or intermediate distances, as is also the case for the majority of known gamma-ray pulsars.

From the radio detections of PSR J0002+6216 and PSR J0631+0646 we could determine DM values and use the NE2001 model to extract the DM distances given in Table 5. For both pulsars the NE2001 distance is very large. The distance for PSR J0002+6216 of $7.7 \mathrm{kpc}$ leads to a gamma-ray efficiency $\eta=L_{\gamma} / \dot{E}$ of about $120 \%$. For PSR J0631+0646 a conversion efficiency of $100 \%$ is found for a distance of about $6.7 \mathrm{kpc}$. The NE2001 model therefore probably underestimates the density of free electrons along the lines of sight to these pulsars. Interestingly, the recently published model for the distribution of free electrons in the Galaxy of Yao et al. (2017) finds DM distances of 6.3 and $4.6 \mathrm{kpc}$ for PSR J0002+6216 and PSR J0631+0646, respectively. The latter distance values lead to realistic efficiency estimates below $100 \%$ (81\% and $90 \%$, respectively).

\subsection{X-Ray Counterpart Searches}

We reanalyzed archival X-ray observations to search for counterparts to the new gamma-ray pulsars and to characterize their X-ray spectra. All our targets except PSR J1827-1446 have adequate coverage by at least one of the major contemporary observatories operating in the soft X-ray band: Swift (Burrows et al. 2005), XMM-Newton (Strüder et al. 2001; Turner et al. 2001), and Chandra (Garmire et al. 2003). The $\mathrm{X}$-ray coverage ranges from few-kilosecond shallow snapshots with Swift to orbit-long, deep observations by Chandra and XMM-Newton. Almost all the detected pulsars have been observed by Swift as part of a systematic survey of the gammaray error boxes of the unidentified Fermi LAT sources (Stroh \& Falcone 2013).

We reduced and analyzed the $X M M-N e w t o n$ data through the most recent release of the XMM-Newton Science Analysis Software (SAS) v15.0. We performed a standard data processing, using the epproc and emproc tools, and screening for high particle background time intervals following Salvetti et al. (2015). For the Chandra data analysis we used the Chandra Interactive Analysis of Observations (CIAO) software version 4.8. We recalibrated event data by using the chandra_repro tool. Swift data were processed and filtered with standard procedures and quality cuts ${ }^{26}$ using FTOOLS tasks in the HEASOFT software package v6.19 and the calibration files in the latest Calibration Database release.

We performed a standard data analysis and source detection in the $0.3-10 \mathrm{keV}$ energy range of the XMM-Newton-EPIC, Chandra-ACIS, and Swift-XRT observations (e.g., Marelli et al. 2015; Salvetti et al. 2015). We preferred the $X M M$ and

\footnotetext{
${ }^{26}$ More detail in http://swift.gsfc.nasa.gov/docs/swift/analysis/.
} 
Table 5

Pulse Shape Parameters and Derived Pulsar Parameters

\begin{tabular}{|c|c|c|c|c|c|c|c|}
\hline PSR & Peaks & $\delta$ & $\Delta$ & Off-pulse Phase Range & $\begin{array}{c}\dot{E} \\
\left(10^{33} \mathrm{erg} \mathrm{s}^{-1}\right)\end{array}$ & $\begin{array}{l}\text { DM Distance } \\
(\mathrm{kpc})\end{array}$ & $\begin{array}{l}\text { Heuristic Distance, } d_{h} \\
(\mathrm{kpc})\end{array}$ \\
\hline $\mathrm{J} 0002+6216$ & 2 & $0.171 \pm 0.011$ & $0.361 \pm 0.012$ & $0.59-1.00$ & 153 & $7.7,6.3$ & 2.0 \\
\hline $\mathrm{J} 0359+5414$ & 1 & $\cdots$ & $\cdots$ & $0.00-0.58$ & 1318 & $\cdots$ & 2.3 \\
\hline $\mathrm{J} 1057-5851$ & 1 & $\ldots$ & $\ldots$ & $0.75-0.24$ & 17 & $\ldots$ & 0.8 \\
\hline J1105-6037 & 2 & $\cdots$ & $0.317 \pm 0.006$ & $0.90-0.38$ & 116 & $\cdots$ & 1.2 \\
\hline $\mathrm{J} 1350-6225$ & 2 & $\cdots$ & $0.485 \pm 0.002$ & $0.92-0.24,0.52-0.77$ & 133 & $\cdots$ & 1.3 \\
\hline $\mathrm{J} 1624-4041$ & 2 & $\cdots$ & $0.429 \pm 0.003$ & $0.44-0.70$ & 39 & $\cdots$ & 1.8 \\
\hline $\mathrm{J} 1650-4601$ & 2 & $\ldots$ & $0.331 \pm 0.005$ & $0.48-1.00$ & 291 & $\ldots$ & 1.1 \\
\hline $\mathrm{J} 1827-1446$ & 2 & $\ldots$ & $0.256 \pm 0.008$ & $0.82-0.32$ & 14 & $\ldots$ & 0.7 \\
\hline $\mathrm{J} 1844-0346$ & 1 & $\cdots$ & $\cdots$ & $0.31-0.92$ & 4249 & $\cdots$ & 2.4 \\
\hline $\mathrm{J} 2017+3625$ & 2 & $\cdots$ & $0.374 \pm 0.004$ & $0.02-0.42,0.58-0.68$ & 12 & $\cdots$ & 0.7 \\
\hline
\end{tabular}

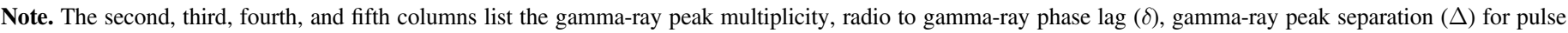

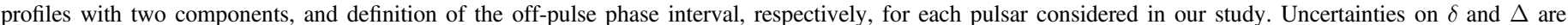

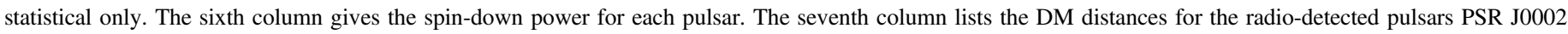

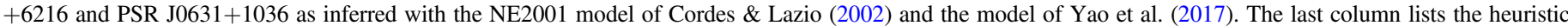
distance, described in Section 4.4.

Chandra observatories if the same field has been observed because of the better performance in terms of effective area and spatial resolution. For each of the X-ray counterparts we performed a spectral analysis using XSPEC v12.9. After extracting response matrices and effective area files, we extracted X-ray fluxes by fitting the spectra with a power-law (PL) model using either a $\chi^{2}$ or the C-statistic (Cash 1979) in the case of low counts $(<100$ photons $)$ and negligible background. For sources characterized by low statistics (typically $\leqslant 30$ photons), we fixed the column density to the value of the Galactic $N_{\mathrm{H}}$ integrated along the line of sight (Dickey \& Lockman 1990) and scaled for the heuristic distance, and, if necessary, we set the X-ray PL photon index $\left(\Gamma_{X}\right)$ to 2 . All quoted uncertainties on the spectral parameters are reported at the $1 \sigma$ confidence level. For each pulsar we computed the corresponding gamma-ray-to-X-ray flux ratio. As reported in Marelli et al. (2015), this could give important information on the nature of the detected pulsar. Finally, for all undetected ones, we computed the $3 \sigma$ X-ray detection limit based on the measured signal-to-noise ratio, assuming a PL spectrum with $\Gamma_{\mathrm{X}}=2$ and the integrated Galactic $N_{\mathrm{H}}$, scaled for the heuristic distance. The detailed results of these analyses are reported in Table 9.

Out of the 13 gamma-ray pulsars, we detected a significant X-ray counterpart for six. PSR J0002+6216, PSR J1105 -6037, and PSR J1844-0344 were detected with Swift-XRT. These sources are listed in the First Swift-XRT Point Source (1SXPS) Catalog (Evans et al. 2014) as 1SXPS J000257.6 +621609, 1SXPS J110500.3-603713, and 1SXPS J184432.9 -034626 , respectively. These sources are located at $(\alpha, \delta)$ $(\mathrm{J} 2000)=\left(0^{\circ} .7404,+62^{\circ} .2692\right),\left(166^{\circ} .2515,-60^{\circ} .6203\right)$, and $\left(281^{\circ} .1371,-3^{\circ} .7740\right)$ with $90 \%$ confidence error circles of $4 !{ }^{\prime \prime} 9,6$." 4, and 2"'7. Owing to the long Chandra exposure time, we clearly detected both the pulsar and the associated nebula of PSR J0359+5414. The pulsar is located at $(\alpha, \delta)=\left(59^{\circ} .8586\right.$, $\left.+54^{\circ} .2486\right)$ with a $90 \%$ confidence error circle of $1^{\prime \prime}$. The nebula is approximately elliptical, with semimajor and semiminor axes of $\sim 15^{\prime \prime}$ and $\sim 7^{\prime \prime}$, respectively, roughly centered on the pulsar position. The nebula is well fitted by an absorbed PL model with photon index equal to $1.4 \pm 0.2$ and unabsorbed flux in the $0.3-10 \mathrm{keV}$ energy band of $(1.3 \pm 0.3) \times 10^{-14} \mathrm{erg} \mathrm{cm}^{-2} \mathrm{~s}^{-1}$. We also detected the counterpart for PSR $\mathrm{J} 2017+3625$ at $(\alpha, \delta)=\left(304^{\circ} .4827\right.$, $36^{\circ} 4189$ ), with a $2^{\prime \prime}$ error, from analysis of a Chandra observation. From XMM-Newton data we detected two possible counterparts for PSR J1624-4041 at 13" from the gamma-ray pulsar position. The two plausible X-ray counterparts are located at $(\alpha, \delta)=\left(246^{\circ} .0372,-40^{\circ} .6931\right)$ and $\left(246^{\circ} .0459,-40^{\circ} .6899\right)$, both with a $0 . " 8$ statistical plus $1 . " 5$ systematic error. We report both counterparts in Table 9, as $s r c 1$ and $s r c 2$, respectively.

\section{Discussion}

A total of 17 gamma-ray pulsars have been discovered among the 118 3FGL sources we have selected for the search, based on their gamma-ray emission properties being suggestive of pulsar emission. The high discovery rate of about $15 \%$ is comparable to that of previous similar surveys, of $\sim 8 \%-12 \%$ (Abdo et al. 2009; Pletsch et al. 2012a, 2013), even though we are searching fainter and fainter LAT sources. The improved semi-coherent blind search technique, the new Pass 8 LAT data, and the improved source selection and localization likely played an important role in the success of the survey. It is interesting to note that a number of sources in our list had already been searched for pulsations in the past. For example, comparing the sky locations searched in our survey with those analyzed in previous Einstein@Home or Atlas surveys (Pletsch et al. 2012a, 2013), we find that about 27\% (32/118) of our sources had already been searched, and 11 of these have now been found to be gamma-ray pulsars. The multiple improvements in our new gamma-ray blind survey enumerated above likely explain the detections of these pulsars. Similarly, seven of the new discovered pulsars (PSR J0002+6216, PSR J0631 +0646, PSR J1035-6720, PSR J1057-5851, PSR J1105 -6037, PSR J1623-5005, and PSR J1624-4041) fall below the sensitivity limit of the previously used search algorithm (see Section 5.1 of Paper I for more details). 
Table 6

Definition of Radio Observation Codes

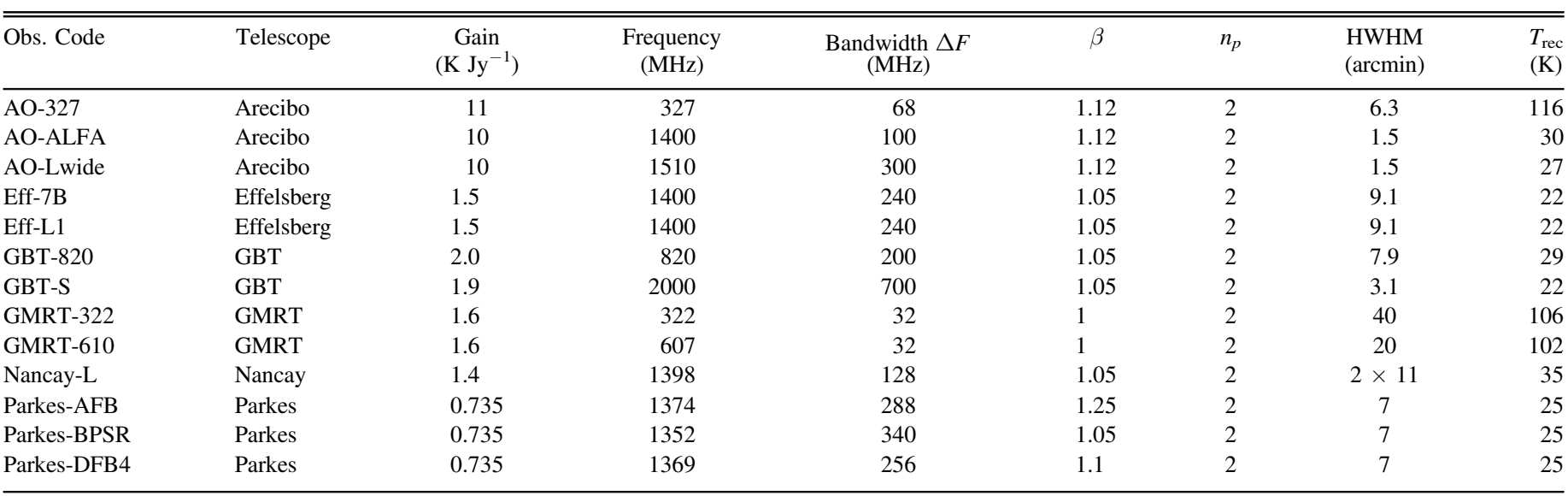

Note. Radio telescopes and backend parameters used for follow-up observations of the new pulsars, described in Section 4.2.

Comparing our target list with the best pulsar candidates from Saz Parkinson et al. (2016), who also used machine-learning techniques for classifying 3FGL unassociated sources, we find a relatively high overlap of about $60 \%$. Interestingly, PSR J0631 +0646 and PSR J1827-1446, discovered in our survey, do not appear in their list. In the case of PSR J0631+0646 this could be caused by the possible association with a nearby SNR, while for PSR J1827-1446 the source detection significance of $\sim 9.1 \sigma$ is simply under the $10 \sigma$ threshold set by Saz Parkinson et al. (2016) for constructing their list. The good overlap between the two target lists makes us confident that we have selected and searched 3FGL sources likely powered by unknown pulsars.

The spectral properties (photon indices $\Gamma$ and cutoff energies $\left.E_{\text {cut }}\right)$ for the surveyed sources, for the 13 new gamma-ray pulsars, and for pulsars from the $2 \mathrm{PC}$ catalog are displayed in Figure 3. The photon indices and cutoff energies of the new Einstein@Home pulsars are very similar to those of 2PC pulsars, a natural consequence of the source selection procedure described in Section 2.2. This is confirmed by a Kolmogorov-Smirnov test, which finds a $\sim 90 \%$ probability that the two samples are drawn from the same parent distribution. The GMM algorithm used for classifying 3FGL sources therefore seems to have efficiently selected pulsar candidates among unassociated sources, which is further supported by the fact that $\sim 80 \%$ of the discovered pulsars were found in the top half of Table 1 . The gamma-ray fluxes of the new pulsars are generally lower than those of 2PC pulsars found in blind searches, also unsurprisingly.

Possible reasons for the nondetections of pulsars in other 3FGL unassociated sources listed in Table 1 are that these sources could be pulsars with low pulse fractions or broad gamma-ray pulse profiles, for which the sensitivity of the search algorithm is lower (see Section 3 of Paper I for a detailed discussion of the search sensitivity). They could also be pulsars with high timing noise, or they could be millisecond pulsars in binary systems. A number of sources in our list were indeed recently identified as candidate binary millisecond Pulsars after we started our search, for instance, 3FGL J0212.1 +5320 (Li et al. 2016), 3FGL J0744.1-2523 (Salvetti et al. 2017), and 3FGL J2039.6-5618 (Salvetti et al. 2015). The discovery of pulsars in binary systems in gamma rays requires initial guesses of the orbital parameters, from, e.g., optical or X-ray observations (see, e.g., Pletsch et al. 2012b). If all searched sources are indeed gamma-ray pulsars, then we would expect a good number of them to be in binaries, based on the 2PC pulsar population.

As can be seen from Figure 3 and Table 1, a number of sources included in our survey had $\Gamma$ and/or $E_{\text {cut }}$ parameters higher than those of 2PC pulsars. These sources were selected by the GMM based on their low variability and moderate curvature indices. Although the gamma-ray emission properties of these sources seem different from those of 2PC pulsars a priori, we included them in the survey for completeness but failed to find new pulsars in any of them. One possibility for the future would be to train the GMM not to select these peculiar sources, or to continue searching in order not to miss pulsars with large spectral index and/or cutoff values. In any case, half of the searched sources from Table 1 have $\Gamma$ and $E_{\text {cut }}$ parameters resembling those of $2 \mathrm{PC}$ pulsars and are thus still prime targets for pulsation searches.

Of the 13 new pulsars reported in this article, only two have been detected in radio. The deep radio follow-up observations conducted as part of this project placed tight constraints on the flux densities of the undetected pulsars. Only six young pulsars among the 54 discovered in blind searches of the LAT data have so far been detected as radio pulsars. The many nondetections in radio are not surprising, given that past radio pulsar surveys have covered the entire sky with moderate sensitivity (see, e.g., Cordes et al. 2006; Keith et al. 2010; Barr et al. 2013; Boyles et al. 2013; Deneva et al. 2013). PSR J0002 +6216 and PSR J0631+0646, both detected in radio, are, however, perfect examples of pulsars with low radio flux densities that would be missed in the short integration times of traditional radio pulsar surveys. The LAT was therefore crucial for the discovery of all these young pulsars, and blind search surveys are clearly key for completing the population of young and energetic gamma-ray pulsars. The discovery of these 13 pulsars with Einstein@Home brings the total number of nonrecycled gamma-ray pulsars to 112 , of which $\sim 54 \%$ are radio-loud. The fraction thus remains similar to that reported in 2PC. As the Fermi mission continues, it will be interesting to see how this fraction evolves, as it is a powerful discriminant of pulsar emission models. 
Table 7

Radio Search Observations of the New Pulsars

\begin{tabular}{|c|c|c|c|c|c|c|c|c|}
\hline $\begin{array}{l}\text { Target } \\
\text { PSR }\end{array}$ & Obs. Code & Date & $\begin{array}{c}t_{\mathrm{int}} \\
\text { (minutes) }\end{array}$ & $\begin{array}{l}\text { R.A. } \\
\text { (J2000) }\end{array}$ & $\begin{array}{l}\text { Decl. } \\
\text { (J2000) }\end{array}$ & $\begin{array}{c}\text { Offset } \\
\text { (arcmin) }\end{array}$ & $\begin{array}{l}T_{\text {sky }} \\
(\mathrm{K})\end{array}$ & $\begin{array}{c}S_{\min } \\
(\mu \mathrm{Jy})\end{array}$ \\
\hline \multirow[t]{2}{*}{$\mathrm{J} 0002+6216$} & GBT-S & 2013 Feb 28 & 28 & 00:02:40.3 & $62: 16: 44.0$ & 2.2 & 0.9 & Detected \\
\hline & Eff-L1 & 2015 Feb 14 & 120 & 00:02:58.1 & $62: 16: 09.6$ & 0.0 & 2.4 & Detected \\
\hline \multirow[t]{5}{*}{$\mathrm{J} 0359+5414$} & Eff-7B & 2010 May 25 & 32 & 03:59:35.8 & $54: 10: 40.8$ & 4.5 & 2.0 & 34 \\
\hline & Eff-7B & $2010 \mathrm{Jul} 17$ & 60 & 03:59:31.5 & $54: 11: 44.1$ & 3.3 & 2.0 & 23 \\
\hline & GBT-S & 2012 Nov 17 & 40 & 03:59:36.3 & $54: 12: 56.5$ & 2.5 & 0.8 & 18 \\
\hline & GBT-S & 2013 Mar 17 & 7 & $03: 59: 36.3$ & $54: 12: 56.5$ & 2.5 & 0.8 & 42 \\
\hline & Eff-L1 & 2015 Feb 14 & 115 & 03:59:26.0 & $54: 14: 55.6$ & 0.0 & 2.0 & 15 \\
\hline \multirow[t]{3}{*}{$\mathrm{J} 0631+0646$} & Eff-L1 & 2015 Feb 13 & 120 & $06: 31: 52.4$ & $06: 46: 15.3$ & 0.0 & 1.8 & Detected \\
\hline & AO-327 & 2015 Mar 15 & 75 & $06: 31: 52.4$ & $06: 46: 14.0$ & 0.0 & 78.4 & Detected \\
\hline & AO-Lwide & 2015 Jun 14 & 69 & $06: 31: 52.4$ & $06: 46: 14.0$ & 0.0 & 1.5 & Detected \\
\hline \multirow[t]{2}{*}{$\mathrm{J} 1057-5851$} & Parkes-DFB4 & 2015 Aug 05 & 70 & 10:57:09.3 & $-58: 51: 11.0$ & 0.1 & 3.9 & 49 \\
\hline & Parkes-DFB4 & 2015 Aug 06 & 51 & $10: 57: 09.3$ & $-58: 51: 11.0$ & 0.1 & 3.9 & 58 \\
\hline \multirow[t]{2}{*}{ J1105-6037 } & Parkes-DFB4 & 2015 Aug 05 & 70 & 11:05:00.5 & $-60: 37: 15.6$ & 0.0 & 5.7 & 52 \\
\hline & Parkes-DFB4 & 2015 Aug 06 & 60 & 11:05:00.5 & $-60: 37: 15.6$ & 0.0 & 5.7 & 56 \\
\hline \multirow[t]{4}{*}{$\mathrm{J} 1350-6225$} & Parkes-AFB & 2010 Nov 19 & 145 & $13: 49: 36.0$ & $-62: 24: 00.0$ & 8.1 & 10.5 & 110 \\
\hline & Parkes-BPSR & 2010 Nov 19 & 144 & $13: 49: 36.0$ & $-62: 24: 00.0$ & 8.1 & 10.9 & 86 \\
\hline & Parkes-DFB4 & 2015 Sep 05 & 433 & $13: 50: 44.5$ & $-62: 25: 43.7$ & 0.0 & 10.6 & 24 \\
\hline & Parkes-DFB4 & 2015 Sep 13 & 500 & $13: 50: 44.5$ & $-62: 25: 43.7$ & 0.0 & 10.6 & 23 \\
\hline \multirow[t]{2}{*}{$\mathrm{J} 1623-5005$} & Parkes-BPSR & 2010 Nov 19 & 144 & $16: 22: 48.0$ & $-50: 06: 00.0$ & 2.7 & 16.9 & 45 \\
\hline & Parkes-AFB & 2010 Nov 19 & 88 & $16: 22: 48.0$ & $-50: 06: 00.0$ & 2.7 & 16.2 & 74 \\
\hline \multirow[t]{9}{*}{ J1624-4041 } & Parkes-AFB & 2009 Dec 02 & 120 & $16: 24: 06.2$ & $-40: 40: 48.0$ & 1.0 & 4.1 & 41 \\
\hline & GBT-S & 2009 Dec 23 & 30 & $16: 24: 06.0$ & $-40: 40: 48.0$ & 1.0 & 1.5 & 15 \\
\hline & Parkes-AFB & 2010 Jul 18 & 120 & $16: 24: 03.0$ & $-40: 42: 56.0$ & 1.9 & 4.1 & 43 \\
\hline & Parkes-AFB & 2010 Jul 26 & 120 & 16:24:03.0 & $-40: 42: 56.0$ & 1.9 & 4.1 & 43 \\
\hline & Parkes-AFB & 2010 Nov 12 & 60 & $16: 24: 03.0$ & $-40: 42: 56.0$ & 1.9 & 4.1 & 60 \\
\hline & GMRT-610 & 2011 Feb 15 & 60 & $16: 24: 03.8$ & $-40: 41: 20.4$ & 1.2 & 34.4 & 297 \\
\hline & Parkes-AFB & $2012 \mathrm{Jul} 12$ & 60 & 16:24:09.0 & $-40: 40: 23.0$ & 1.1 & 4.1 & 58 \\
\hline & GMRT-322 & 2012 Jul 12 & 60 & $16: 24: 09.0$ & $-40: 40: 23.0$ & 1.1 & 178.6 & 618 \\
\hline & Parkes-AFB & 2012 Dec 17 & 60 & $16: 24: 09.0$ & $-40: 40: 23.0$ & 1.1 & 4.1 & 58 \\
\hline \multirow[t]{2}{*}{$\mathrm{J} 1650-4601$} & Parkes-BPSR & 2010 Nov 21 & 144 & $16: 50: 48.0$ & $-46: 06: 00.0$ & 6.9 & 14.4 & 74 \\
\hline & Parkes-AFB & 2010 Nov 21 & 139 & $16: 50: 48.0$ & $-46: 06: 00.0$ & 6.9 & 13.8 & 96 \\
\hline \multirow[t]{2}{*}{$\mathrm{J} 1827-1446$} & GBT-820 & 2014 Apr 21 & 35 & $18: 27: 20.2$ & $-14: 46: 01.2$ & 1.2 & 33.2 & 60 \\
\hline & Eff-L1 & 2015 Feb 14 & 120 & $18: 27: 24.6$ & $-14: 46: 25.4$ & 0.0 & 8.3 & 19 \\
\hline \multirow[t]{5}{*}{$\mathrm{J} 1844-0346$} & Eff-7B & 2010 May 15 & 32 & $18: 44: 15.4$ & $-03: 42: 46.8$ & 5.7 & 11.8 & 53 \\
\hline & Eff-7B & $2010 \mathrm{Jul} 30$ & 60 & $18: 44: 21.8$ & $-03: 42: 03.6$ & 5.2 & 11.8 & 37 \\
\hline & Eff-7B & $2010 \mathrm{Jul} 31$ & 60 & $18: 44: 21.8$ & $-03: 42: 03.6$ & 5.2 & 11.8 & 37 \\
\hline & GBT-S & 2012 Nov 17 & 22 & $18: 44: 26.2$ & $-03: 45: 21.6$ & 2.0 & 4.7 & 24 \\
\hline & Eff-7B & 2015 Aug 27 & 120 & 18:44:33.0 & $-03: 46: 32.0$ & 0.0 & 11.8 & 21 \\
\hline \multirow[t]{10}{*}{$\mathrm{J} 2017+3625$} & Nancay-L & 2010 May 05 & 65 & $20: 17: 55.8$ & $36: 25: 08.0$ & 0.0 & 4.6 & 50 \\
\hline & Nancay-L & 2010 May 11 & 47 & $20: 17: 55.8$ & $36: 25: 08.0$ & 0.0 & 4.6 & 58 \\
\hline & GBT-S & 2010 May 13 & 60 & 20:17:59.0 & $36: 25: 19.0$ & 0.7 & 1.8 & 10 \\
\hline & GBT-820 & 2011 Jan 15 & 45 & $20: 17: 57.6$ & $36: 27: 36.0$ & 2.5 & 18.6 & 43 \\
\hline & AO-ALFA & 2015 May 11 & 20 & $20: 17: 54.2$ & $36: 23: 24.0$ & 1.8 & 4.6 & 34 \\
\hline & AO-327 & 2015 Jun 24 & 15 & 20:17:55.9 & $36: 27: 32.4$ & 2.4 & 202.7 & 170 \\
\hline & AO-327 & 2015 Jun 25 & 15 & 20:17:55.9 & $36: 27: 32.4$ & 2.4 & 202.7 & 170 \\
\hline & Eff-7B & 2015 Aug 27 & 120 & $20: 17: 55.8$ & $36: 25: 08.0$ & 0.0 & 4.6 & 17 \\
\hline & AO-327 & 2015 Nov 16 & 28 & 20:17:55.9 & $36: 25: 08.4$ & 0.0 & 202.7 & 113 \\
\hline & AO-Lwide & 2015 Nov 17 & 33 & $20: 17: 55.9$ & $36: 25: 08.4$ & 0.0 & 3.8 & 5 \\
\hline
\end{tabular}

Note. Radio observations of the new pulsars. In the cases of PSR J0002+6216 and PSR J0631+0646, radio pulsations were detected (see Section 4.2). 
Table 8

Light-curve Modeling Results

\begin{tabular}{|c|c|c|c|c|c|c|c|c|c|c|}
\hline PSR & $\mathrm{TPC}-\ln \mathcal{L}$ & $\begin{array}{r}\text { TPC } \alpha \\
\text { (deg) }\end{array}$ & $\begin{array}{r}\text { TPC } \zeta \\
(\operatorname{deg})\end{array}$ & $\operatorname{TPC} f_{\Omega}$ & TPC Radio Flag & $\mathrm{OG}-\ln \mathcal{L}$ & $\begin{array}{r}\text { OG } \alpha \\
(\operatorname{deg})\end{array}$ & $\begin{array}{l}\text { OG } \zeta \\
(\operatorname{deg})\end{array}$ & $\mathrm{OG} f_{\Omega}$ & OG Radio Flag \\
\hline $\mathrm{J} 0002+6216$ & 110.26 & $64_{-2}^{+3}$ & $54 \pm 2$ & $1.05 \pm 0.04$ & $\ldots$ & 105.70 & $69_{-1}^{+8}$ & $58_{-1}^{+25}$ & $1.08_{-0.27}^{+0.05}$ & $\cdots$ \\
\hline $\mathrm{J} 0359+5414^{\dagger}$ & 39.88 & $1 \pm 1$ & $2 \pm 1$ & $19.62_{-8.52}^{+0.01}$ & $\mathrm{~L}$ & 38.04 & $80_{-6}^{+8}$ & $24 \pm 4$ & $1,01_{-0.41}^{+0.09}$ & Q \\
\hline $\mathrm{J} 1105-6037$ & 46.11 & $61_{-27}^{+4}$ & $49_{-7}^{+21}$ & $0.98_{-0.31}^{+0.05}$ & $\mathrm{~F}$ & 67.40 & $8_{-2}^{+5}$ & $71_{-1}^{+4}$ & $0.99_{-0.09}^{+0.01}$ & Q \\
\hline $\mathrm{J} 1350-6225$ & 79.42 & $82_{-4}^{+2}$ & $85_{-2}^{+1}$ & $0.82 \pm 0.10$ & $\mathrm{~L}$ & 48.16 & $90 \pm 9$ & $88_{4}^{+1}$ & $0.70 \pm 0.03$ & L \\
\hline J1624-4041 & 86.57 & $71_{-5}^{+2}$ & $58_{-5}^{+1}$ & $1.13 \pm 0.03$ & $\mathrm{~F}$ & 72.90 & $86 \pm 1$ & $68 \pm 1$ & $1.02_{-0.01}^{+0.02}$ & $\mathrm{~F}$ \\
\hline $\mathrm{J} 1650-4601$ & 46.30 & $13_{-7}^{+2}$ & $69 \pm 1$ & $0.47_{-0.09}^{+0.01}$ & Q & 54.13 & $11_{-4}^{+2}$ & $74_{-3}^{+6}$ & $0.21_{-0.16}^{+0.19}$ & Q \\
\hline $\mathrm{J} 1827-1446^{\dagger}$ & 52.65 & $1 \pm 1$ & $2 \pm 1$ & $69.16_{-5.67}^{+0.01}$ & $\mathrm{~L}$ & 45.04 & $75_{-11}^{+1}$ & $26_{-1}^{+5}$ & $1.34_{-0.71}^{+0.01}$ & Q \\
\hline $\mathrm{J} 1844-0346^{\dagger}$ & 23.06 & $10 \pm 1$ & $68 \pm 1$ & $0.49 \pm 0.07$ & Q & 22.08 & $79_{-4}^{+6}$ & $22_{-3}^{+1}$ & $0.99_{-0.39}^{+0.31}$ & Q \\
\hline $\mathrm{J} 2017+3625$ & 168.10 & $23 \pm 5$ & $69 \pm 1$ & $0.52_{-0.01}^{+0.16}$ & Q & 127.47 & $16_{-5}^{+12}$ & $80_{-5}^{+1}$ & $0.23_{-0.04}^{+0.10}$ & Q \\
\hline
\end{tabular}

Note. Light-curve fitting results for all pulsars except PSR J0631+0646. The first column gives the pulsar name; a $\dagger$ indicates that the $\Delta \ln \mathcal{L}$ surface was not renormalized. The second (seventh) column gives the best-fit $-\ln \mathcal{L}$ value for the TPC (OG) model. The third, fourth, and fifth (eight, ninth, and 10th) columns give the best-fit $\alpha$ and $\zeta$ with corresponding $f_{\Omega}$ for the TPC (OG) model. For pulsars without a radio detection, the sixth (11th) column gives a radio-loudness prediction from the best-fit geometry for the TPC $(\mathrm{OG})$ model: $\mathrm{L}=$ radio-loud, $\mathrm{F}=$ radio-faint, and $\mathrm{Q}=$ radio-quiet; see the text for details.
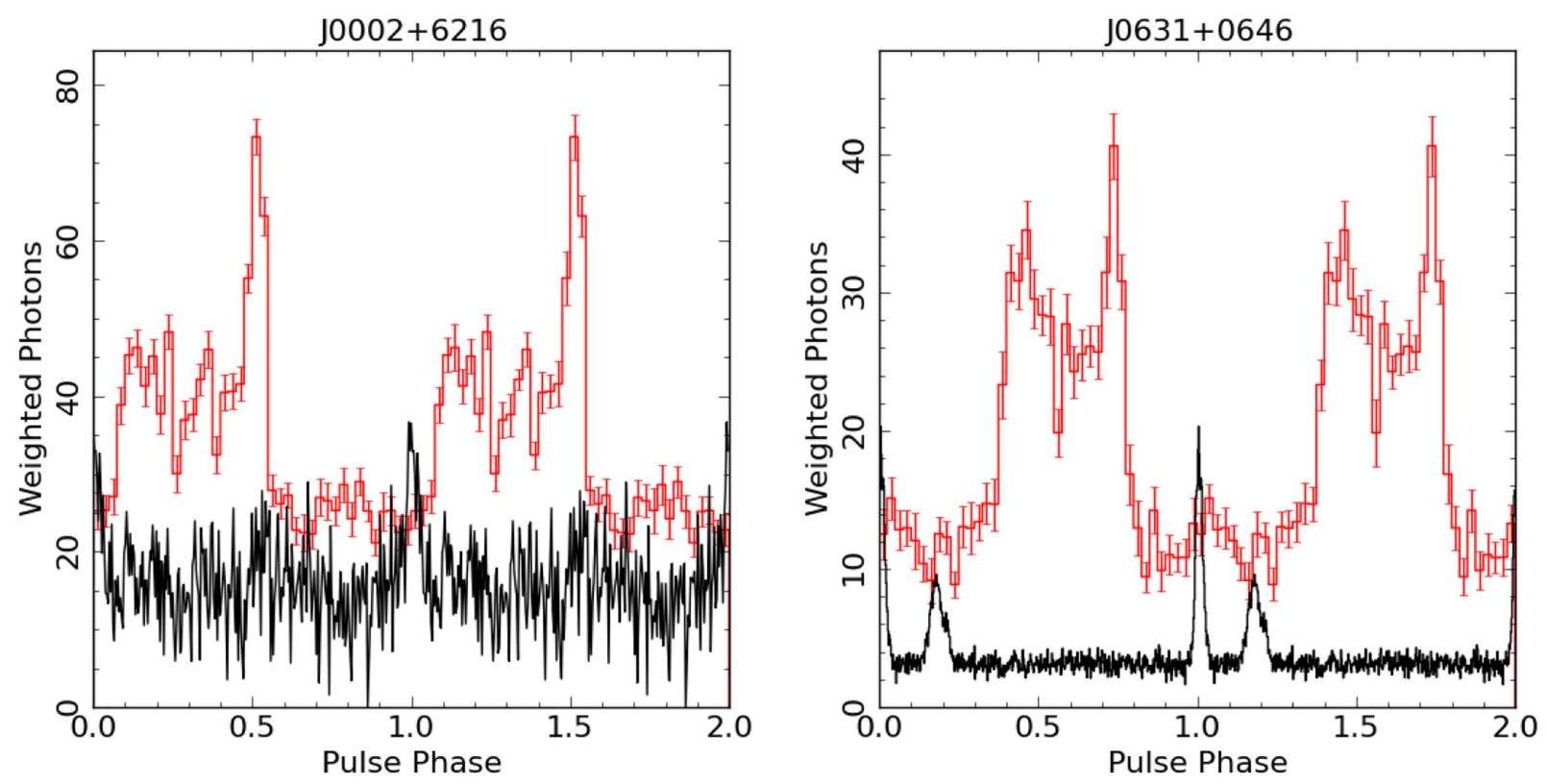

Figure 4. Radio and gamma-ray pulse profiles for PSR J0002+6216 (left) and PSR J0631+0646 (right). Two complete cycles are shown for clarity. Weighted LAT gamma-ray pulse profiles (in red) were produced by selecting photons with weights greater than 0.05 . Radio profiles (in black) correspond to $1.4 \mathrm{GHz}$ observations made with the Effelsberg telescope for PSR J0002+6216 and the Arecibo telescope for PSR J0631+0646. Uncertainties in DM converted to uncertainties in the phase offset between the radio and gamma-ray peaks correspond to $\sim 1 \%$ of the rotational periods.

Recently, Fermi LAT Collaboration (2017) released a catalog of resolved point sources in a $40^{\circ} \times 40^{\circ}$ region around the Galactic center direction. By selecting spectrally curved sources and comparing the SEDs of these point sources with those of a large sample of 3FGL sources, they could identify pulsar-like candidates from these new Galactic bulge sources. These sources are also prime targets for future blind pulsation searches.

\section{Conclusions}

Using information from a preliminary version of the 3FGL catalog of Fermi LAT sources, we have selected 118 targets with pulsar-like emission properties. We produced Pass 8 LAT data sets for each of the sources, and these data sets were then searched for pulsations with a multistage blind search algorithm, utilizing the volunteer computing system Einstein@Home. This survey led to the discovery of 17 pulsars, of which 13 are presented in this article, and the other pulsars have been or will be published elsewhere.

On-pulse and off-pulse gamma-ray spectral analyses were conducted for each of the new pulsars. The gamma-ray emission properties of the 13 newly discovered pulsars reported in this paper are similar to those of other young gamma-ray pulsars, such as those from the 2PC catalog. Radio follow-up observations were carried out, resulting in the detections of two 
Table 9

Summary of the Pulsar X-Ray Spectral Parameters

\begin{tabular}{|c|c|c|c|c|c|c|}
\hline PSR & $\begin{array}{c}\text { X-ray } \\
\text { Observatory }^{\mathrm{a}}\end{array}$ & $\begin{array}{c}\text { Exposure } \\
(\mathrm{ks})\end{array}$ & $\begin{array}{c}N_{\mathrm{H}} \\
\left(10^{21} \mathrm{~cm}^{-2}\right)\end{array}$ & $\Gamma_{X}$ & $\begin{array}{c}F_{X}^{\mathrm{b}} \\
\left(10^{-14} \mathrm{erg} \mathrm{cm}^{-2} \mathrm{~s}^{-1}\right)\end{array}$ & $\overline{G_{100} / F_{X}^{\mathrm{c}}}$ \\
\hline $\mathrm{J} 0002+6216$ & Swift & 9.2 & $1.0^{\mathrm{c}}$ & $2^{\mathrm{c}}$ & $4.3_{-2.4}^{+1.9}$ & $600_{-200}^{+760}$ \\
\hline $\mathrm{J} 0359+5414$ & Chandra & 29.7 & $2.0 \pm 1.1$ & $2.7 \pm 0.3$ & $0.96 \pm 0.20$ & $5800 \pm 1500$ \\
\hline J0631+0646 & Swift & 3.5 & $0.4^{\mathrm{c}}$ & $2^{\mathrm{c}}$ & $<9.1$ & $>400$ \\
\hline J1057-5851 & Chandra & 10.1 & $3.0^{\mathrm{c}}$ & $2^{\mathrm{c}}$ & $<0.25$ & $>20000$ \\
\hline J1105-6037 & Swift & 16 & $1.0^{\mathrm{c}}$ & $2^{\mathrm{c}}$ & $4.8_{-1.4}^{+1.9}$ & $1300_{-380}^{+550}$ \\
\hline $\mathrm{J} 1350-6225$ & Swift & 5.4 & $1.4^{\mathrm{c}}$ & $2^{\mathrm{c}}$ & $<8.1$ & $>740$ \\
\hline J1528-5838 & Swift & 6 & $0.7^{\mathrm{c}}$ & $2^{\mathrm{c}}$ & $<6.2$ & $>480$ \\
\hline $\mathrm{J} 1623-5005$ & XMM-Newton & 85.4 & $4.0^{\mathrm{c}}$ & $2^{\mathrm{c}}$ & $<2.0$ & $>4100$ \\
\hline \multirow[t]{2}{*}{ J1624-4041 } & XMM-Newton & 31.0 & $2.0^{\mathrm{c}}$ & $(\mathrm{src} 1) 0.7 \pm 0.2$ & $3.7 \pm 0.7$ & $430 \pm 100$ \\
\hline & & & & $(\operatorname{src} 2) 2.0 \pm 0.4$ & $1.0 \pm 0.3$ & $1600 \pm 690$ \\
\hline $\mathrm{J} 1650-4601$ & Swift & 3.5 & $1.0^{\mathrm{c}}$ & $2^{\mathrm{d}}$ & $<10.8$ & $>1100$ \\
\hline J1827-1446 & $\ldots$ & $\ldots$ & $\ldots$ & $\cdots$ & $\ldots$ & $\ldots$ \\
\hline J1844-0344 & Swift & 82 & $2.4^{\mathrm{c}}$ & $2^{\mathrm{c}}$ & $7.6 \pm 1.3$ & $1300 \pm 260$ \\
\hline $\mathrm{J} 2017+3625$ & Chandra & 10.0 & $1.0^{\mathrm{c}}$ & $2^{\mathrm{c}}$ & $1.7 \pm 0.7$ & $3600 \pm 2600$ \\
\hline
\end{tabular}

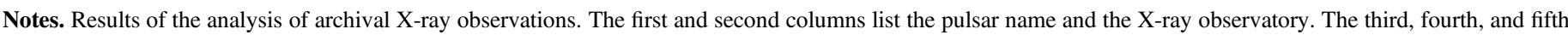

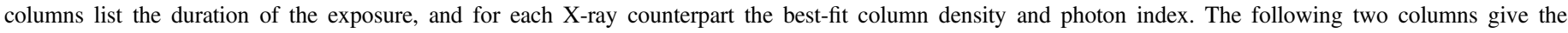
unabsorbed X-ray flux in the $0.3-10 \mathrm{keV}$ energy band and the gamma-ray-to-X-ray flux ratio. All uncertainties are reported at the $68 \%$ confidence level.

${ }^{a}$ We report only the X-ray observatory used for the spectral analysis.

b When the X-ray counterpart is not detected, we report the minimum X-ray unabsorbed flux required for a $3 \sigma$ detection.

${ }^{c}$ Gamma-ray energy fluxes in $0.1-100 \mathrm{GeV}$ are used to calculate the gamma-ray-to-X-ray flux ratio.

d Due to the low statistics in these sources, we fixed this parameter in the spectral analysis as described in the text.

of them with low radio flux densities. The pulse profiles of the 13 new pulsars were fit using the TPC and OG models. For some of the pulsars, radio emission is predicted by the models but is still undetected in follow-up or archival observations.

The increased sensitivity of the blind search algorithm, the improved Pass 8 LAT data, and improved source selection and relocalization pipeline enabled us to maintain a relatively high detection rate, compared to previous similar surveys. Nevertheless, for a number of the 3FGL sources with clear pulsar-like properties selected for the search, we were unable to find a pulsar. These sources remain excellent targets for future searches. New systematic surveys such as the one presented in this paper and in Paper I are warranted, and so are blind searches for millisecond pulsars in binary systems, which at the moment can only be searched using external constraints on the orbital parameters from observations at other wavelengths.

This work was supported by the Max-Planck-Gesellschaft (MPG), by the Deutsche Forschungsgemeinschaft(DFG) through an Emmy Noether research grant PL 710/1-1 (PI: Holger J. Pletsch), and by NSF award 1104902.

The authors also gratefully acknowledge the support of the many thousands of Einstein@Home volunteers, without whom this search would not have been possible.

The Fermi LAT Collaboration acknowledges generous ongoing support from a number of agencies and institutes that have supported both the development and the operation of the LAT, as well as scientific data analysis. These include the National Aeronautics and Space Administration and the Department of Energy in the United States; the Commissariat à l'Energie Atomique and the Centre National de la Recherche Scientifique/Institut National de Physique Nucléaire et de Physique des Particules in France; the Agenzia Spaziale Italiana and the Istituto Nazionale di Fisica Nucleare in Italy; the Ministry of Education, Culture, Sports, Science and Technology (MEXT), High Energy Accelerator Research Organization (KEK) and Japan Aerospace Exploration Agency (JAXA) in Japan; and the
K. A. Wallenberg Foundation, the Swedish Research Council, and the Swedish National Space Board in Sweden.

Additional support for science analysis during the operations phase is gratefully acknowledged from the Istituto Nazionale di Astrofisica in Italy and the Centre National d'Études Spatiales in France. This work performed in part under DOE contract DE-AC02-76SF00515.

Fermi research at NRL is supported by NASA. The Parkes radio telescope is part of the Australia Telescope, which is funded by the Commonwealth Government for operation as a National Facility managed by CSIRO. We thank our colleagues for their assistance with the radio timing observations. The Robert C. Byrd Green Bank Telescope (GBT) is operated by the National Radio Astronomy Observatory, a facility of the National Science Foundation operated under cooperative agreement by Associated Universities, Inc. The Arecibo Observatory is operated by SRI International under a cooperative agreement with the National Science Foundation (AST-1100968), and in alliance with Ana G. Méndez-Universidad Metropolitana and the Universities Space Research Association. The Nançay Radio Observatory is operated by the Paris Observatory, associated with the French Centre National de la Recherche Scientifique (CNRS). The Effelsberg $100 \mathrm{~m}$ telescope is operated by the MPIfR (Max-Planck-Institut für Radioastronomie). We acknowledge support of GMRT operators. The GMRT is run by the National Centre for Radio Astrophysics of the Tata Institute of Fundamental Research.

\section{ORCID iDs}

J. Wu (iD https://orcid.org/0000-0003-3536-4368

C. J. Clark (1D) https://orcid.org/0000-0003-4355-3572

H. J. Pletsch (iD https://orcid.org/0000-0002-1164-4755

L. Guillemot (iD https://orcid.org/0000-0002-9049-8716

D. J. Champion (iD https://orcid.org/0000-0003-1361-7723

J. Deneva (i) https://orcid.org/0000-0003-1226-0793

P. S. Ray (i) https://orcid.org/0000-0002-5297-5278

M. Kramer (iD https://orcid.org/0000-0002-4175-2271

C. Aulbert (iD https://orcid.org/0000-0002-1481-8319 
C. Beer (1) https://orcid.org/0000-0003-3991-067X

F. Camilo (i) https://orcid.org/0000-0002-1873-3718

H. B. Eggenstein (1) https://orcid.org/0000-0001-5296-7035

M. Kerr (1) https://orcid.org/0000-0002-0893-4073

S. M. Ransom (1) https://orcid.org/0000-0001-5799-9714

\section{References}

Abdo, A. A., Ackermann, M., Ajello, M., et al. 2009, Sci, 325, 840 Abdo, A. A., Ackermann, M., Ajello, M., et al. 2010, ApJ, 711, 64 Abdo, A. A., Ajello, M., Allafort, A., et al. 2013, ApJS, 208, 17 Acero, F., Ackermann, M., Ajello, M., et al. 2015, ApJS, 218, 23 Acero, F., Ackermann, M., Ajello, M., et al. 2016a, ApJS, 223, 26 Acero, F., Ackermann, M., Ajello, M., et al. 2016b, ApJS, 224, 8 Allafort, A., Baldini, L., Ballet, J., et al. 2013, ApJL, 777, L2 Atwood, W., Albert, A., Baldini, L., et al. 2013, arXiv:1303.3514 Atwood, W. B., Abdo, A. A., Ackermann, M., et al. 2009, ApJ, 697, 1071 Barr, E. D., Guillemot, L., Champion, D. J., et al. 2013, MNRAS, 429, 1633 Bhattacharyya, B., Roy, J., Ray, P. S., et al. 2013, ApJL, 773, L12 Boyles, J., Lynch, R. S., Ransom, S. M., et al. 2013, ApJ, 763, 80 Burrows, D. N., Hill, J. E., Nousek, J. A., et al. 2005, SSRv, 120, 165 Camilo, F., Kerr, M., Ray, P. S., et al. 2012, ApJ, 746, 39 Camilo, F., Kerr, M., Ray, P. S., et al. 2015, ApJ, 810, 85 Camilo, F., Ray, P. S., Ransom, S. M., et al. 2009, ApJ, 705, 1 Cash, W. 1979, ApJ, 228, 939

Cheng, K. S., Ho, C., \& Ruderman, M. 1986, ApJ, 300, 500 Clark, C. J., Pletsch, H. J., Wu, J., et al. 2015, ApJL, 809, L2

Clark, C. J., Pletsch, H. J., Wu, J., et al. 2016, ApJL, 832, L15 Clark, C. J., Wu, J., Pletsch, H. J., et al. 2017, ApJ, 834, 106

Cognard, I., Guillemot, L., Johnson, T. J., et al. 2011, ApJ, 732, 47

Cordes, J. M., Freire, P. C. C., Lorimer, D. R., et al. 2006, ApJ, 637, 446

Cordes, J. M., \& Lazio, T. J. W. 2002, arXiv:astro-ph/0207156

Cromartie, H. T., Camilo, F., Kerr, M., et al. 2016, ApJ, 819, 34

de Jager, O. C., Raubenheimer, B. C., \& Swanepoel, J. W. H. 1989, A\&A, 221,180

Deneva, J. S., Stovall, K., McLaughlin, M. A., et al. 2013, ApJ, 775, 51

Deutsch, A. J. 1955, AnAp, 18, 1

Dickey, J. M., \& Lockman, F. J. 1990, ARA\&A, 28, 215

Dyks, J., \& Rudak, B. 2003, ApJ, 598, 1201

Evans, P. A., Osborne, J. P., Beardmore, A. P., et al. 2014, ApJS, 210, 8

Fermi LAT Collaboration 2017, arXiv:1705.00009

Garmire, G. P., Bautz, M. W., Ford, P. G., Nousek, J. A., \& Ricker, G. R., Jr. 2003, Pproc. SPIE, 4851, 28

Guillemot, L., Freire, P. C. C., Cognard, I., et al. 2012a, MNRAS, 422, 1294

Guillemot, L., Johnson, T. J., Venter, C., et al. 2012b, ApJ, 744, 33

Guillemot, L., Kramer, M., Johnson, T. J., et al. 2013, ApJ, 768, 169
Haslam, C. G. T., Salter, C. J., Stoffel, H., \& Wilson, W. E. 1982, A\&AS, 47,1

Hewish, A., Bell, S. J., Pilkington, J. D. H., Scott, P. F., \& Collins, R. A. 1968, Natur, 217, 709

Johnson, T. J., Venter, C., Harding, A. K., et al. 2014, ApJS, 213, 6

Kalapotharakos, C., Harding, A. K., \& Kazanas, D. 2014, ApJ, 793, 97

Kalapotharakos, C., Harding, A. K., Kazanas, D., \& Contopoulos, I. 2012, ApJL, 754, L1

Keith, M. J., Jameson, A., van Straten, W., et al. 2010, MNRAS, 409, 619

Keith, M. J., Johnston, S., Ray, P. S., et al. 2011, MNRAS, 414, 1292

Kerr, M. 2010, PhD thesis, Univ. Washington

Kerr, M. 2011, ApJ, 732, 38

Kerr, M., Camilo, F., Johnson, T. J., et al. 2012, ApJL, 748, L2

Lee, K. J., Guillemot, L., Yue, Y. L., Kramer, M., \& Champion, D. J. 2012, MNRAS, 424, 2832

Li, K.-L., Kong, A. K. H., Hou, X., et al. 2016, ApJ, 833, 143

Lorimer, D., \& Kramer, M. 2005, Handbook of Pulsar Astronomy (Cambridge: Cambridge Univ. Press)

Maan, Y. 2015, ApJ, 815, 126

Maan, Y., Aswathappa, H. A., \& Deshpande, A. A. 2012, MNRAS, 425, 2

Marelli, M., Mignani, R. P., De Luca, A., et al. 2015, ApJ, 802, 78

Mignani, R. P., Paladino, R., Rudak, B., et al. 2017, ApJL, submitted (arXiv: 1708.02828 )

Mirabal, N., Frías-Martinez, V., Hassan, T., \& Frías-Martinez, E. 2012, MNRAS, 424, L64

Muslimov, A. G., \& Harding, A. K. 2003, ApJ, 588, 430

Muslimov, A. G., \& Harding, A. K. 2004, ApJ, 606, 1143

Pierbattista, M., Harding, A. K., Grenier, I. A., et al. 2015, A\&A, 575, A3

Pletsch, H. J., Guillemot, L., Allen, B., et al. 2012a, ApJ, 744, 105

Pletsch, H. J., Guillemot, L., Allen, B., et al. 2013, ApJL, 779, L11

Pletsch, H. J., Guillemot, L., Fehrmann, H., et al. 2012b, Sci, 338, 1314

Ransom, S. M., Ray, P. S., Camilo, F., et al. 2011, ApJL, 727, L16

Salvetti, D., Mignani, R. P., De Luca, A., et al. 2015, ApJ, 814, 88

Salvetti, D., Mignani, R. P., De Luca, A., et al. 2017, MNRAS, 470, 466

Saz Parkinson, P. M., Dormody, M., Ziegler, M., et al. 2010, ApJ, 725 571

Saz Parkinson, P. M., Xu, H., Yu, P. L. H., et al. 2016, ApJ, 820, 8

Scargle, J. D., Norris, J. P., Jackson, B., \& Chiang, J. 2013, ApJ, 764, 167

Story, S. A., Gonthier, P. L., \& Harding, A. K. 2007, ApJ, 671, 713

Stroh, M. C., \& Falcone, A. D. 2013, ApJS, 207, 28

Strüder, L., Briel, U., Dennerl, K., et al. 2001, A\&A, 365, L18

Swiggum, J. K., Kaplan, D. L., McLaughlin, M. A., et al. 2017, ApJ, 847,25

Turner, M. J. L., Abbey, A., Arnaud, M., et al. 2001, A\&A, 365, L27

Venter, C., Harding, A. K., \& Guillemot, L. 2009, ApJ, 707, 800

Watters, K. P., Romani, R. W., Weltevrede, P., \& Johnston, S. 2009, ApJ, 695, 1289

Yao, J. M., Manchester, R. N., \& Wang, N. 2017, ApJ, 835, 29 